\title{
Semidefinite Relaxation and Approximation Analysis of a Beamformed Alamouti Scheme for Relay Beamforming Networks
}

\author{
Sissi Xiaoxiao Wu, Anthony Man-Cho So, Jiaxian Pan and Wing-Kin Ma
}

\begin{abstract}
In this paper, we study the amplify-and-forward (AF) schemes in two-hop one-way relay networks. In particular, we consider the multigroup multicast transmission between long-distance users. Given that perfect channel state information is perceived, our goal is to design the AF process so that the max-min-fair (MMF) signal-to-interference-plus-noise ratio (SINR) is optimized subject to generalized power constraints. We propose a rank-two beamformed Alamouti (BFA) AF scheme and formulate the corresponding AF design problem as a two-variable fractional quadratically-constrained quadratic program (QCQP), which is further tackled by the semidefinite relaxation (SDR) technique. We analyze the approximation quality of two-variable fractional SDRs under the Gaussian randomization algorithm. These results are fundamentally new and reveal that the proposed BFA AF scheme can outperform the traditional BF AF scheme, especially when there are many users in the system or many generalized power constraints in the problem formulation. From a practical perspective, the BFA AF scheme offers two degrees of freedom (DoFs) in beamformer design, as opposed to the one DoF offered by the BF AF scheme, to improve the receivers' SINR. In the latter part of this paper, we demonstrate how this extra DoF leads to provable performance gains by considering two special cases of multicasting, where the AF process is shown to employ a special structure. The numerical simulations further validate that the proposed BFA AF scheme outperforms the BF AF scheme and works well for large-scale relay systems.
\end{abstract}

Index terms - MIMO relay network, distributed relay network, cognitive radio, energy harvesting, amplify-and-forward (AF), multigroup multicast, SDR, approximation bounds.

EDICS: SPC-PERF (Performance analysis and bounds) MSP-APPL (Applications of MIMO communications and signal processing), SAM-BEAM (Applications of sensor and array multichannel processing)

This work was supported in part by the Hong Kong Research Grant Council (RGC) General Research Fund (GRF) Project CUHK 416012, and in part by The Chinese University of Hong Kong Direct Grant No. 2050506.

Sissi Xiaoxiao Wu is the corresponding author. She is now with the Signal, Information, Networks and Energy Laboratory, Arizona State University, Tempe, United States. E-mail: xxwu.eesissi@gmail.com. Anthony Man-Cho So is with the Department of Systems Engineering and Engineering Management, CUHK, Hong Kong. E-mail: manchoso@ee.cuhk.edu.hk. Jiaxian Pan and Wing-Kin Ma are with the Department of Electronic Engineering, The Chinese University of Hong Kong (CUHK), Hong Kong. E-mail: jxpan@ee.cuhk.edu.hk, wkma@ieee.org. 


\section{INTRODUCTION}

The information delivery between multiple wireless devices has shown an increasing importance in up-to-date military networks, relay networks, and 5G networks [1] -[4]]. The state-ofthe-art technique in this context is to use small smart access points (APs), e.g., mobile phones, wireless relays, Wi-Fi APs, to assist information delivery between far-apart transceiver pairs. Nowadays, a new trend is to connect the smart APs by fibers, microwave or millimeter wave to build up a cloud processing center for facilitating reliable communications. A typical example is the cloud radio access network (C-RAN) [5]-[7], which is recently proposed as a promising network architecture to offer a 1000x increase in capacity to support broadband applications. The key enabling technologies in C-RANs are the cloud processors pool and fronthaul-backhaul links. They coordinate all the base-stations in all cells as a cloud base-station and serve the users in a jointly optimized way. In view of this, it is promising to extend the "cloud relays" by considering the communications between devices as information delivery in a relay network. We treat the intra-network interference as noise and try to handle the inter-network interference by designing the AF process in the cloud center. This gives rise to the so-called cloud relay network (C-RN) [3], [8]-[10]; see a system model example in Figure 1]

In this work, we are interested in a typical two-hop one-way relay network. In particular, we consider the case that all the nodes, i.e., the transmitter, the receiver, and relays, are equipped with a single antenna. This assumption is reasonable, as nodes in a D2D communication network are usually limited by power and apparatus. In our setting, the transmitter and receiver are farapart, and thus they rely on the relays to amplify and forward (AF) the information. 1] since the direct link between them are negligible. We assume that the relays are distributively located, and more importantly, they coordinate to form a C-RN. In practice, the capacity of the fronthaulbackhaul links in C-RNs is an important issue. If the link capacity is unlimited, both the channel state information (CSI) and received signals can be shared within the cloud, and thus the system becomes an MIMO relay network. On the other hand, if the link capacity is limited in such a way that only CSIs are shared and the received signals are isolated among different relays, then the system is reduced to a distributed relay network. We remark that there are also other kinds of relay networks corresponding to different link capacities, but in this paper we focus on the

\footnotetext{
${ }^{1}$ The relays can also decode-and-forward (DF) the received signals, but this is beyond the scope of this paper.
} 
two above.

In this paper, we are interested in relay $\mathrm{AF}$ design for multigroup multicast transmission. In the literature, there are different problem formulations; see, e.g., [11]-[19]. Herein, we focus on the max-min-fair (MMF) formulation, in which the worst user's signal-to-interference-plusnoise ratio (SINR) is to be maximized under generalized power constraints, such as total power constraint, per-relay power constraints, interference temperature constraints, or energy harvesting constraints. This makes our design approach applicable to many scenarios. A classic approach for AF relays is to adopt the beamformed (BF) AF scheme [11], [12], which gives rise to an NPhard one-variable fractional quadratically-constrained quadratic program (QCQP). An efficient way to tackle QCQP is to apply the semidefinite relaxation (SDR) technique [20]. That is, we first rewrite the fractional QCQP to a rank-one constrained fractional semidefinite program (SDP). Then, by dropping the non-convex rank-one constraint, we solve the SDP in polynomial time. It is well known that the SDR is tight if the corresponding SDP has rank-one solutions. Otherwise, a Gaussian randomization algorithm is applied to convert the SDP optimal solution into a feasible solution to the fractional QCQP [21], [22]. We shall call this solution the SDR solution in the sequel. A fundamental issue here is to quantify the quality of the SDR solution. In our previous work [9], [10], we show that the SDP always has a rank-one solution when $M+J \leq 4$, and that the SINR associated with the SDR solution is at least $\Omega\left(\frac{1}{M \log J}\right)$ times that associated with the optimal solution to the fractional QCQP when $M+J>4$, where $M$ is the number of users (receivers) in the network and $J$ is the number of generalized power constraints. This result provides an SDR approximation bound for the one-variable fractional QCQP when multiple constraints are present. However, from a practical perspective, it actually implies that the SINR associated with the SDR-based BF AF scheme may experience a performance loss on the order of $1 /(M \log J)$ in large-scale systems.

In order to improve the relay beamforming performance, we propose to adopt the Alamouti space-time code in the AF structure. This leads to the BF Alamouti (BFA) AF scheme, in which two beamformers are used to process two data symbols jointly. Compared to the BF AF scheme, which uses only one beamformer to process a single data symbol, the BFA AF scheme has one extra degree of freedom (DoF) in the beamformer design. As such, it is expected that the latter will yield better system performance. In fact, the extra DoF available to the BFA AF scheme is also manifested in its corresponding design problem, in that it can be shown to admit a two- 
variable fractional QCQP formulation. Our analytical results show that the optimal value of the corresponding two-variable SDP is always no worse than that of the one-variable SDP. In addition, the SDR is tight when $M+J \leq 5$, and when $M+J>5$, the approximation quality of the SDR-based BFA AF scheme is on the order of $1 /(\sqrt{M} \log J)$. Clearly, both the tightness and approximation quality results are better than their BF AF counterparts.

The idea of using the Alamouti code in the context of single-group multicast beamforming has been introduced in our previous work [23], where the design problem is a standard QCQP. The subsequent conference paper [22] proposed the BFA scheme for multigroup multicasting without relays. This paper significantly improves [22] by generalizing the aforementioned BFA scheme to relay networks with generalized power constraints and providing an analysis on its approximation performance. It is worth mentioning that the authors in [24] also introduced a rank-two BF AF scheme for relay networks. However, the scenario they focused on is single-group multicasting in a distributed relay network with one power constraint, while our work considers multigroup multicasting for both the distributed relay and MIMO relay networks with generalized power constraints. We remark that the generalization from single-group multicasting to multigroup multicasting, and from distributed relays to MIMO relays, are non-trivial. More importantly, our work establishes for the first time the SDR approximation quality of a fairly general class of two-variable fractional QCQPs. It should also be noted that the problem considered in this paper, namely beamformer design for multi-user to multi-user multigroup multicasting in relay networks, has not been well addressed in the literature. Indeed, existing works on relay transceiver design mainly focus on the point-to-point [14], [15], single-user to multi-user [16], multi-user to single-user [17], [18], and multi-user to multi-user unicast [11] and multicast scenarios [19]. The work in [25] studies the rank-two relay multicasting with a direct link, while our work is targeted at far-apart devices without direct links and our scenario setting is the more generalized multigroup multicasting. The work in [26] studies the beamformer design in a multigroup multicast relay network. However, it considers BF AF schemes for single-antenna relays, whereas our focus is on rank-two BFA AF scheme under a cloud relay setting. We remark that some efficient heuristics have recently been proposed to find a high-quality solution to one-variable fractional QCQPs; see, e.g., [27]-[30]. We numerically compare their results with the proposed BF Alamouti AF scheme in the the companion technical report [31], wherein we could see that even though those heuristics can help find a better QCQP solution, the proposed BFA AF still 
owns a significantly better performance.

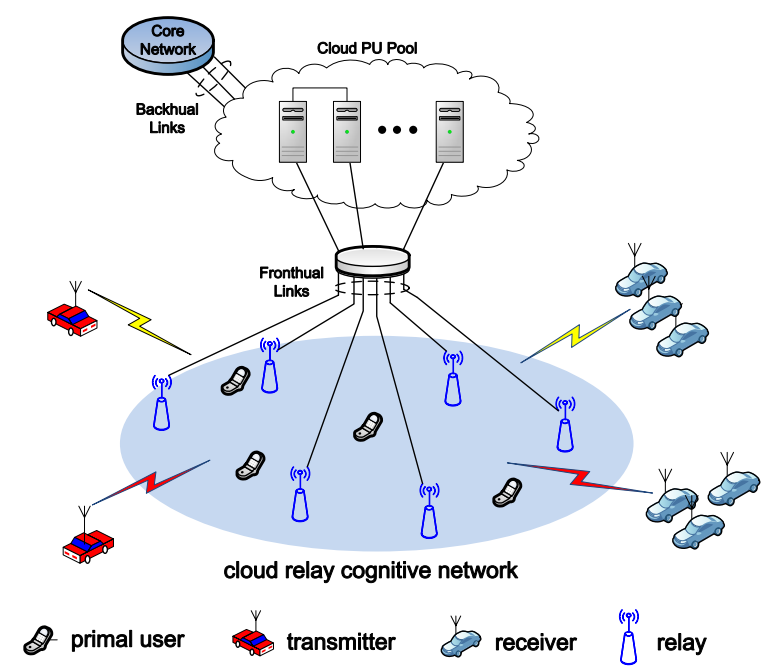

Fig. 1. An example of the cloud relay network.

The organization of this paper is as follows. In Section II, we introduce the system model and review the SDR-based BF AF scheme for both the MIMO relay network and distributed relay network in the presence of primal users. We show how the design problem for the BF AF scheme can be tackled by the SDR technique and introduce the existing approximation bounds on the performance of the SDR solution. In Section III, we introduce the BFA AF scheme and show how the corresponding design problem gives rise to a new two-variable fractional QCQP formulation. As one of our main results, we establish an approximation bound for the SDR of the two-variable fractional QCQP, thereby providing a performance guarantee for the proposed BFA AF scheme. In Section IV, special cases of multicasting relay networks are studied, from which we see how the two DoFs offered by the BFA AF scheme can improve the system performance. Lastly, simulation results are provided in Section $\mathrm{V}$, and the paper is concluded in Section VI.

Our notation is standard: $\mathbb{C}^{N}$ is the set of all complex $N$-dimensional vectors; $\mathbb{H}_{+}^{N}$ is the set of all $N \times N$ positive semidefinite matrices; $\|\cdot\|$ is the vector Euclidean norm; $\boldsymbol{A} \bullet \boldsymbol{B}$ stands for the inner product between matrix $\boldsymbol{A}$ and $\boldsymbol{B} ; \boldsymbol{A} \otimes \boldsymbol{B}$ stands for the Kronecker product between matrix $\boldsymbol{A}$ and $\boldsymbol{B} ; \boldsymbol{A} \odot \boldsymbol{B}$ stands for the element-wise product between matrix $\boldsymbol{A}$ and $\boldsymbol{B} ; \operatorname{vec}(\boldsymbol{A})$ is the vectorization operator for matrix $\boldsymbol{A} ; \operatorname{Diag}(\mathbf{x})$ is a diagonal matrix parametrized with the 
elements of $\mathbf{x} ; \operatorname{rank}(\boldsymbol{X})$ and $\lambda_{\min }(\boldsymbol{X})$ stand for the rank and the smallest eigenvalue, resp.; $\mathbf{e}_{i}$ is a unit vector with the nonzero element at the $i$ th entry; $\boldsymbol{I}_{r}$ denotes the $r$-by- $r$ identity matrix; $\mathbb{E}[\cdot]$ is statistical expectation; $\mathcal{C N}(\mathbf{0}, \boldsymbol{W})$ is used to denote the circularly symmetric complex Gaussian distribution with mean vector $\mathbf{0}$ and covariance matrix $\boldsymbol{W}$.

\section{System Model AND THE BeAmformed AMPLIFy-AND-ForwARd SCHEMES}

In this section, we describe the system model for two-hop one-way relay networks. In particular, we consider the multigroup multicast transmission by a network of single-antenna AF relays. We assume that there are $L$ relays in the network; $G$ single-antenna transmitters (sources) send $G$ independent common information to $G$ groups of single-antenna users (receivers, destinations); users in the same group require the same information while users in different groups require different information. In total there are $\sum_{j=1}^{G} m_{j}=M$ users in the network, where $m_{j}$ is the number of users in group $j, \forall j=1, \ldots, G$. In our target setting, the transmitters and receivers are far-apart such that the direct link between them can be ignored. Thus, relays play an important role during the information delivery by amplifying and forwarding (AF) received signals from sources to destinations. By introducing the cloud relay network $(\mathrm{C}-\mathrm{RN})$ concept [8], we consider that the relays are distributively located but connected by a cloud PUs pool (i.e., the computation center) via fronthaul and backhaul links, which are typically fibers or microwave connected to fibers. We assume that all nodes in the network are well synchronized, channels from transmitters to relays and relays to users are frequency flat, quasi-static, and the channels are perfectly perceived by transmitters sending reference signals and then passed to the PUs pool. The PUs pool can serve as a genie in the network to design the AF process.

\section{A. The Beamformed AF Scheme}

Let us first introduce the traditional AF scheme, i.e., the BF AF scheme, which is widely used in existing relay networks, e.g., [11], [12]. We begin with the information delivery process in a one-way relay network, which is proceed by two hops:

1) Source-to-relay hop: the transmitters send information to relays. The receive model at the relay is written as follows

$$
\mathbf{r}(t)=\sum_{j=1}^{G} \boldsymbol{f}_{j} s_{j}(t)+\mathbf{n}(t)
$$


where $\mathbf{r}(t)=\left[r^{1}(t), \ldots, r^{\ell}(t), \ldots, r^{L}(t)\right]^{T}$ with

$$
r^{\ell}(t)=\sum_{j=1}^{G} f_{j}^{\ell} s_{j}(t)+n^{\ell}(t), \quad \ell=1, \ldots, L
$$

being the received signal at relay $\ell ; s_{j}(t)$ is the common information specific for group $j$ with $\mathbb{E}\left[\left|s_{j}(t)\right|^{2}\right]=P_{j}$, where $P_{j}$ is the transmit power at transmitter $j ; \boldsymbol{f}_{j}=\left[f_{j}^{1}, \ldots, f_{j}^{\ell}, \ldots, f_{j}^{L}\right]^{T}$ is the channel from transmitter $j$ to all the relays; $\mathbf{n}(t)=\left[n^{1}(t), \ldots, n^{\ell}(t), \ldots, n^{L}(t)\right]^{T}$ with $n^{\ell}(t)$ being the Gaussian noise at relay $\ell$ which has mean zero and variance $\sigma_{\ell}^{2}$. Throughout this paper, we assume that $\sigma_{\ell}^{2}>0, \forall \ell$.

2) Relay-to-destination hop: relays process the received signals and then forward them to receivers. In particular, the transmit structure at the relay side can be given by

$$
\mathbf{x}(t)=\boldsymbol{V} \mathbf{r}(t)
$$

The weighting matrix $\boldsymbol{V}$ represents how the relays interact with each other. Specifically, we may consider all the single-antenna relays are connected by a cloud, within which the CSIs are fully shared. Then, if the received signals are shared within the cloud, $\boldsymbol{V}$ could be any arbitrary matrix to be optimized, and we are dealing with an MIMO relay network [12]; if the received signals are not shared, $\boldsymbol{V}$ is a diagonal matrix, and we are dealing with a distributed relay network [11].

Let us first consider the MIMO relay network. The received signal for user- $(k, i)$, i.e., user $i$ in group $k$, is given by

$$
\begin{aligned}
y_{k, i}(t) & =\boldsymbol{g}_{k, i}^{H} \mathbf{x}(t)+v_{k, i}(t) \\
& =\underbrace{\boldsymbol{g}_{k, i}^{H} \boldsymbol{V} \boldsymbol{f}_{k} s_{k}(t)}_{\text {desired signal }}+ \\
& \underbrace{\boldsymbol{g}_{k, i}^{H} \boldsymbol{V}\left(\sum_{m \neq k} \boldsymbol{f}_{m} s_{m}(t)\right)+\boldsymbol{g}_{k, i}^{H} \boldsymbol{V} \mathbf{n}(t)+\mu_{k, i}(t)}_{\text {interference and noise }},
\end{aligned}
$$

where $\boldsymbol{g}_{k, i}=\left[g_{k, i}^{1}, \ldots, g_{k, i}^{\ell}, \ldots, g_{k, i}^{L}\right]^{T}$ is the channel from relays to user- $(k, i)$ and $\mu_{k, i}(t)$ is the Gaussian noise at user- $(k, i)$ with zero mean and variance $\sigma_{k, i}^{2}$. In this paper, we assume that $\sigma_{k, i}^{2}>0, \forall k, i$. Accordingly, the receive $\operatorname{SINR}$ at user- $(k, i)$ can be expressed as

$$
\frac{P_{k}\left|\boldsymbol{g}_{k, i}^{H} \boldsymbol{V} \boldsymbol{f}_{k}\right|^{2}}{\sum_{m \neq k} P_{m}\left|\boldsymbol{g}_{k, i}^{H} \boldsymbol{V} \boldsymbol{f}_{m}\right|^{2}+\boldsymbol{g}_{k, i}^{H} \boldsymbol{V} \boldsymbol{\Sigma} \boldsymbol{V}^{H} \boldsymbol{g}_{k, i}+\sigma_{k, i}^{2}}
$$

where $\boldsymbol{\Sigma}=\operatorname{Diag}\left(\sigma_{1}^{2}, \ldots, \sigma_{\ell}^{2}, \ldots, \sigma_{L}^{2}\right) \succ \mathbf{0}$. 


\section{B. Generalized Power Constraints}

Our goal is to optimize the AF process for the relay networks. Specifically, to design the AF weighting matrix $\boldsymbol{V}$, three design constraints are under our considerations:

1). Total Power Constraints. A natural consideration is that the total transmit power at relays is below a given threshold. In this way, we have the total power constraint given by

$$
\begin{aligned}
\mathbb{E}\left[\|\mathbf{x}(t)\|^{2}\right] & =\operatorname{Tr}\left(\boldsymbol{V}\left(\sum_{j=1}^{G} P_{j} \boldsymbol{f}_{j} \boldsymbol{f}_{j}^{H}+\boldsymbol{\Sigma}\right) \boldsymbol{V}^{H}\right) \\
& =\boldsymbol{w}^{H} \boldsymbol{D}_{0} \boldsymbol{w} \leq \bar{P}_{0},
\end{aligned}
$$

where (6) is obtained by denoting $\boldsymbol{w}=\operatorname{vec}(\boldsymbol{V})$ and using the identity

$$
\operatorname{Tr}\left(\boldsymbol{A}^{H} \boldsymbol{B} \boldsymbol{C D}\right)=\operatorname{vec}(\boldsymbol{A})^{H}\left(\boldsymbol{D}^{T} \otimes \boldsymbol{B}\right) \operatorname{vec}(\boldsymbol{C}),
$$

which is valid for arbitrary complex matrices $\boldsymbol{A}, \boldsymbol{B}, \boldsymbol{C}, \boldsymbol{D}$ of appropriate dimensions. Also, $\bar{P}_{0}$ is the total transmit power budget for all the relays and $\boldsymbol{D}_{0}$ is defined in (11) in Table I.

2). Per-relay Power Constraints. Another common design constraint is subject to the power allowed at each relay, which can be written as

$$
\mathbf{e}_{\ell}^{H} \boldsymbol{V}\left(\sum_{j=1}^{G} P_{j} \boldsymbol{f}_{j} \boldsymbol{f}_{j}^{H}+\boldsymbol{\Sigma}\right) \boldsymbol{V}^{H} \mathbf{e}_{\ell} \leq \bar{P}_{\ell}, \quad \ell=1, \ldots, L,
$$

where $\bar{P}_{\ell}$ is the maximum transmit power allowed at relay $\ell$. Similar to (6), we can further rewrite it to

$$
\boldsymbol{w}^{H} \boldsymbol{D}_{\ell} \boldsymbol{w} \leq \bar{P}_{\ell}, \quad \ell=1, \ldots, L
$$

where $\boldsymbol{D}_{\ell}$ is defined in (12) in Table I.

3). Interference Temperature Constraints. We may also consider a popular design constraint in the cognitive radio (CR) network is to control the interference temperature at primal users [32]-[34]. We consider a CR relay network with $U$ primal users where the interference is simply caused by the AF relays (not the transmitters). Let $\boldsymbol{h}_{u}=\left[h_{u}^{1}, \ldots, h_{u}^{\ell}, \ldots, h_{u}^{L}\right]^{T}$ be the channel from relays to primal user $u, \forall u=1, \ldots, U$. Then the interference temperature power constraints for primal users are given by

$$
\boldsymbol{w}^{H} \boldsymbol{G}_{u} \boldsymbol{w} \leq \eta_{u}, \quad u=1, \ldots, U
$$

where $\eta_{u}$ is the maximum interference allowed at primal user $u, \boldsymbol{G}_{u}$ is defined in (13) in Table I with $\sigma_{u}^{2}>0$ being the noise power at primal user $u$. 


\section{A Unified AF Design Problem}

In this sub-section, as depicted in Fig,2, we show how the beamformer design for different relay networks can be captured by a unified formulation.

1) Problem Formulation for the MIMO CR Relay Network: In light of the SINR expression in (5), we consider the MMF design approach, which maximizes the worst user's SINR, subject to the power constraints. This gives rise to a one-variable fractional QCQP Problem (R1BF) in Table I. Note that we re-define $p_{\ell}$ with $p_{0}=\bar{P}_{0}$ for $\ell=0$ and $p_{\ell}=\bar{P}_{\ell}$ for $\ell=1, \ldots, L$.

2) Problem Formulation for the Distributed CR Relay Network: As for the distributed relays, since the AF weighting matrix here is a diagonal matrix, by a slight abuse of notation, we may re-define $\boldsymbol{w}=\left.\operatorname{Diag}(\boldsymbol{V})\right|^{2}$ and then the design problem for the distributed CR relay network can be written as exactly the same form as that for the MIMO relay, i.e., Problem (R1BF), given that $\mathcal{L}, \boldsymbol{A}_{k, i}, \boldsymbol{C}_{k, i}, \boldsymbol{D}_{\ell}$ and $\boldsymbol{G}_{u}$ are defined in (14), (15), (16), (17), (18), and (19) in Table I] Note that herein the beamformer $\boldsymbol{w}$ is defined differently from that in the MIMO relay network.

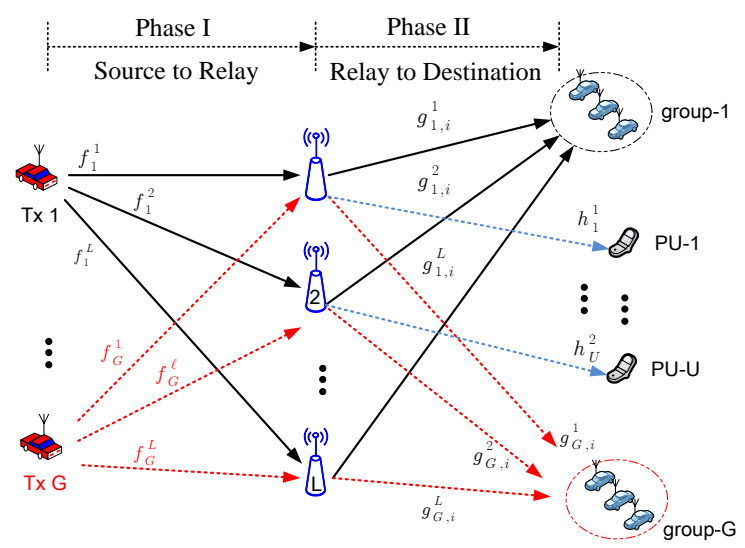

Fig. 2. The two-hop one-way relay network with primal users.

3) Problem Formulation for Other One-hop Relay Networks: Given that the way of interaction between relays is different, we may formulate different AF problems for design purposes. In fact, we could define $\boldsymbol{w}=\operatorname{vec}(\boldsymbol{V}) \in \mathcal{V}$ where $\mathcal{V}$ specifies the prescribed possible structure of the AF matrix. One possible scenario is that the relays are partitioned into several groups, and

\footnotetext{
${ }^{2}$ Note that $\boldsymbol{V}$ is a diagonal matrix in the distributed relay network.
} 
the ones within the same group can fully communicate while the inter-group message passing is with limited capacity. This is typical when the backhaul is not powerful enough to support a massive information sharing. In this paper, we only focus on the MIMO relay network and the distributed relay network and provide a comprehensive performance analysis for them.

TABLE I

Summary OF THE DESIGN PROBlem FOR BF AF SCHEMES

$$
\begin{aligned}
& \text { (R1BF) } \quad \boldsymbol{w}^{\star}=\arg \max _{\boldsymbol{w} \in \mathbb{C}^{\mathcal{L}}} \min _{\substack{k=1, \ldots, G ; \\
i=1, \ldots, m_{k}}} \frac{\boldsymbol{w}^{H} \boldsymbol{A}_{k, i} \boldsymbol{w}}{\boldsymbol{w}^{H} \boldsymbol{C}_{k, i} \boldsymbol{w}+1} \\
& \text { subject to } \quad \boldsymbol{w}^{H} \boldsymbol{D}_{\ell} \boldsymbol{w} \leq \bar{P}_{\ell}, \quad \forall \ell=0,1, \ldots, L, \\
& \boldsymbol{w}^{H} \boldsymbol{G}_{u} \boldsymbol{w} \leq \eta_{u}, \quad \forall u=1, \ldots, U . \\
& \text { (R1SDR) } \quad \boldsymbol{W}^{\star}=\arg \max _{\boldsymbol{W} \in \mathbb{H}_{+}^{\mathcal{C}}} \frac{\boldsymbol{A}_{k, i} \bullet \boldsymbol{W}}{\boldsymbol{C}_{k, i} \bullet \boldsymbol{W}+1} \\
& \text { subject to } \quad \boldsymbol{D}_{\ell} \bullet \boldsymbol{W} \leq \bar{P}_{\ell}, \quad \forall \ell=0,1, \ldots, L, \\
& \boldsymbol{G}_{u} \bullet \boldsymbol{W} \leq \eta_{u}, \quad \forall u=1, \ldots, U . \\
& \mathcal{L}=L^{2} \\
& \boldsymbol{A}_{k, i}=P_{k}\left(\boldsymbol{f}_{k}^{*} \otimes \boldsymbol{g}_{k, i}\right)\left(\boldsymbol{f}_{k}^{*} \otimes \boldsymbol{g}_{k, i}\right)^{H} / \sigma_{k, i}^{2} \\
& \boldsymbol{C}_{k, i}=\sum_{m \neq k} P_{m}\left(\boldsymbol{f}_{m}^{*} \otimes \boldsymbol{g}_{k, i}\right)\left(\boldsymbol{f}_{m}^{*} \otimes \boldsymbol{g}_{k, i}\right)^{H} / \sigma_{k, i}^{2} \\
& +\boldsymbol{\Sigma} \otimes\left(\boldsymbol{g}_{k, i} \boldsymbol{g}_{k, i}\right)^{H} / \sigma_{k, i}^{2} \\
& \boldsymbol{D}_{0}=\left(\sum_{j=1}^{G} P_{j}\left(\boldsymbol{f}_{j}^{*}\right)\left(\boldsymbol{f}_{j}^{*}\right)^{H}+\boldsymbol{\Sigma}\right) \otimes \boldsymbol{I} \\
& \boldsymbol{D}_{\ell}=\left(\sum_{j=1}^{G} P_{j}\left(\boldsymbol{f}_{j}^{*}\right)\left(\boldsymbol{f}_{j}^{*}\right)^{H}+\boldsymbol{\Sigma}\right) \otimes\left(\mathbf{e}_{\ell} \mathbf{e}_{\ell}^{H}\right) \\
& \boldsymbol{G}_{u}=\sum_{j=1}^{G} P_{j}\left(\boldsymbol{f}_{j}^{*} \otimes \boldsymbol{h}_{u}\right)\left(\boldsymbol{f}_{j}^{*} \otimes \boldsymbol{h}_{u}\right)^{H} / \sigma_{u}^{2} \\
& \mathcal{L}=L \\
& \boldsymbol{A}_{k, i}=P_{k}\left(\boldsymbol{f}_{k}^{*} \odot \boldsymbol{g}_{k, i}\right)\left(\boldsymbol{f}_{k}^{*} \odot \boldsymbol{g}_{k, i}\right)^{H} / \sigma_{k, i}^{2} \\
& \boldsymbol{C}_{k, i}=\sum_{m \neq k} P_{m}\left(\boldsymbol{f}_{m}^{*} \odot \boldsymbol{g}_{k, i}\right)\left(\boldsymbol{f}_{m}^{*} \odot \boldsymbol{g}_{k, i}\right)^{H} / \sigma_{k, i}^{2} \\
& +\operatorname{Diag}\left(\left|g_{k, i}^{1}\right|^{2} \sigma_{1}^{2},\left|g_{k, i}^{2}\right|^{2} \sigma_{2}^{2}, \ldots,\left|g_{k, i}^{L}\right|^{2} \sigma_{L}^{2}\right) / \sigma_{k, i}^{2} \\
& \boldsymbol{D}_{0}=\sum_{j=1}^{G} P_{j} \operatorname{Diag}\left(\left(\boldsymbol{f}_{j}^{*}\right)\left(\boldsymbol{f}_{j}^{*}\right)^{H}\right)+\boldsymbol{\Sigma} \\
& \boldsymbol{D}_{\ell}=\left(\sum_{j=1}^{G} P_{j} \operatorname{Diag}\left(\left(\boldsymbol{f}_{j}^{*}\right)\left(\boldsymbol{f}_{j}^{*}\right)^{H}\right)+\boldsymbol{\Sigma}\right) \odot\left(\mathbf{e}_{\ell} \mathbf{e}_{\ell}^{H}\right) \\
& \boldsymbol{G}_{u}=\sum_{j=1}^{G} P_{j}\left(\boldsymbol{f}_{j}^{*} \odot \boldsymbol{h}_{u}\right)\left(\boldsymbol{f}_{j}^{*} \odot \boldsymbol{h}_{u}\right)^{H} / \sigma_{u}^{2}
\end{aligned}
$$

\section{The SDR Technique and Approximation Bounds for One-variable QCQPS}

From preceding discussions, a generalized fractional QCQP problem (R1BF) is formulated for both the MIMO relay network and the distributed relay network. It is well known that Problem (R1BF) is in general NP-hard [11], [20]-[22]. A classic way to tackle this NP-hard problem is to apply the SDR technique [20], which reformulates QCQP as a rank constrained SDP problem 
and then drops the non-convex rank constraint. Specifically, to put SDR into the context, we use the equivalence $\boldsymbol{W}=\boldsymbol{w} \boldsymbol{w}^{H} \Longleftrightarrow \boldsymbol{W} \succeq \mathbf{0}, \operatorname{rank}(\boldsymbol{W}) \leq 1$ to relax (R1BF) to (R1SDR) as shown in Table If It is well known that (R1SDR) can be approximated to arbitrary accuracy by the bisection method [21], [22], where at each iteration of the bisection, an SDP problem is solved in polynomial time [35]. Let us define

$$
\gamma(\mathbf{W})=\min _{\substack{k=1, \ldots, G ; \\ i=1, \ldots, m_{k}}} \frac{\boldsymbol{A}_{k, i} \bullet \boldsymbol{W}}{\boldsymbol{C}_{k, i} \bullet \boldsymbol{W}+1}
$$

and $\boldsymbol{W}^{\star}$ and $\boldsymbol{w}^{\star}$ be the optimal solutions to (R1SDR) and (R1BF), respectively. It follows that $\gamma\left(\boldsymbol{W}^{\star}\right) \geq \gamma\left(\boldsymbol{w}^{\star}\left(\boldsymbol{w}^{\star}\right)^{H}\right)$, since (R1SDR) is a convex relaxation of (R1BF). The equality holds when (R1SDR) has rank-one solutions. If $\operatorname{rank}\left(\boldsymbol{W}^{\star}\right)>1$, a feasible but generally sub-optimal solution $\widehat{\boldsymbol{w}}$ is extracted from $\boldsymbol{W}^{\star}$ by a Gaussian randomization algorithm [21], [22], [36]; more specifically, see Algorithm 1 in [10]. It is easy to see that $\gamma\left(\boldsymbol{w}^{\star}\left(\boldsymbol{w}^{\star}\right)^{H}\right) \geq \gamma\left(\widehat{\boldsymbol{w}}(\widehat{\boldsymbol{w}})^{H}\right)$. We are now interested in determining whether a reverse inequality (approximately) holds, as such an inequality will reveal the approximation quality of the SDR solution. We summarize the prior results in [10], [21] as follows.

Proposition 1 Let $M \geq 1$ be the total number of users in the relay network and $J$ denote the total number of constraints in $(R I B F)$, i.e., $J=L+U+1$ and $J \geq 1$. Then,

a). When $M+J \leq 4,(R l S D R)$ always produces an optimal solution to $(R I B F)$.

b). When $M+J>4$, let $\widehat{\boldsymbol{w}}$ be the solution returned by Gaussian randomization algorithm and $N$ be the number of randomizations. Then, with probability at least $1-(5 / 6)^{N}$, we have

$$
\gamma\left(\widehat{\boldsymbol{w}} \widehat{\boldsymbol{w}}^{H}\right)=\Omega\left(\frac{1}{M \log J}\right) \gamma\left(\boldsymbol{w}^{\star} \boldsymbol{w}^{\star H}\right) .
$$

Proposition 1 implies that the SDR-based BF AF scheme is optimal when $M+J \leq 4$. However, it will experience an SINR performance degradation when $M$ or $J$ is large. Specifically, in the worst case, the approximate quality is on the order of $1 /(M \log J)$. Therefore, it may not work well in large-scale systems.

Remark 1: Recently, there is flourishing interest in using RF wave to transfer power to those users in the system who aim at receiving energy rather than information. We usually call this kind of users as energy receivers (ERs). As in the case with primal users, we could impose $R$ harvested energy constraints to the design problem [37]-[39]. In this case, Proposition 1a) 
still holds by setting $J=L+U+R+1$. However, a similar result to Proposition 1b) holds only when $R=1$. This statement can be proven in a similar manner as Proposition 1 by using Theorem 2 in [40].

\section{The Rank-Two Beamformed Alamouti AF Scheme in the Relay Networks}

Our discussions in Section II reveal that BF AF may experience serious performance degradation in large-scale systems. However, in practice, the multigroup multicast transmission aims to serve groups of users and also, each of the relay should have its own power budget, not to mention that there may be a number of primal users if we consider the CR system. Therefore, the inherent feature of the relay network motivates us to propose more advanced AF schemes, for which the guaranteed performance can scale better with the problem size. We observe in Proposition 1 that the performance degradation results from using a rank-one AF weight $\boldsymbol{w}$ to approximate a high rank SDP solution $\boldsymbol{W}^{\star}$. To remedy this defect, an intuition is to explore one more DoF by introducing the Alamouti space-time code. This motivates the development of the BFA AF scheme.

\section{A. The Beamformed Alamouti AF Scheme for the MIMO relay}

To introduce the Alamouti space-time code structure in the AF process, we parse the transmit signal in every two time slots as a group, i.e., $\mathbf{s}(m)=[s(2 m) s(2 m+1)]^{T}$, and transmit $\mathbf{s}(m)$ in each source-to-relay hop. Specifically, following (3), for any $p=1,2$, we denote the AF weighting matrix at time slot $p$ as $\boldsymbol{V}_{p}=\left[\boldsymbol{v}_{1}^{p}, \ldots, \boldsymbol{v}_{\ell}^{p}, \ldots \boldsymbol{v}_{L}^{p}\right]$ where $\boldsymbol{v}_{\ell}^{p}=\left[v_{1, \ell}^{p}, \ldots, v_{\ell, \ell}^{p}, \ldots, v_{L, \ell}^{p}\right]^{T}$. Then, the transmit signal at relay $\ell$ (for every two time slots) can be modified to

$$
\begin{aligned}
\boldsymbol{X}_{\ell}(m) & =\left[\begin{array}{ll}
X_{\ell}(2 m) & X_{\ell}(2 m+1)
\end{array}\right] \\
& =\sum_{c=1}^{L}\left[v_{\ell, c}^{1}, v_{\ell, c}^{2}\right] \mathbf{C}\left(\mathbf{r}^{c}(m)\right)
\end{aligned}
$$

where $\mathbf{C}: \mathbb{C}^{2} \rightarrow \mathbb{C}^{2 \times 2}$ is the Alamouti space-time code, e.g., $\mathbf{C}(\boldsymbol{x})=\left[\begin{array}{cc}x_{1} & x_{2} \\ -x_{2}^{*} & x_{1}^{*}\end{array}\right]$, and we denote $\mathbf{r}^{\ell}(m)=\left[r^{\ell}(2 m) r^{\ell}(2 m+1)\right]^{T}$ with $r^{\ell}(2 m)$ defined in (2). To demonstrate the signal structure, we illustrate the transmit signal at each relay in Figure 3 . The corresponding receive 


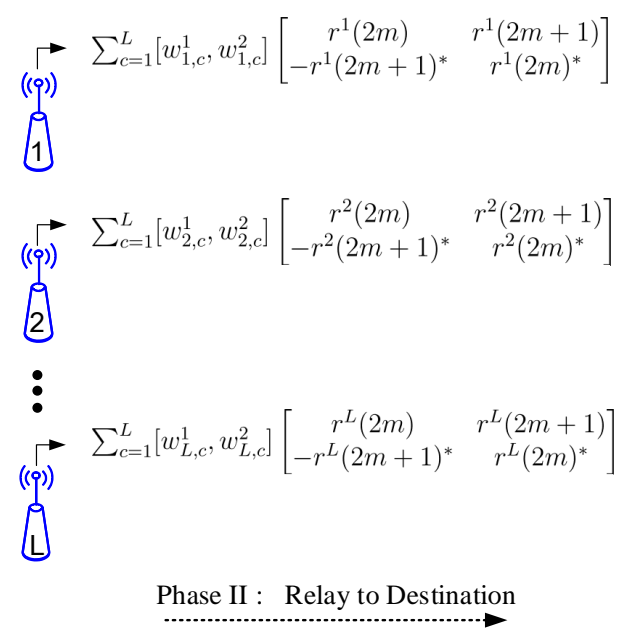

Fig. 3. The BFA AF signal structure for the MIMO relay.

signal at user- $(k, i)$ is given in 20).

$$
\begin{aligned}
& \mathbf{y}_{k, i}(m) \\
& =\left[y_{k, i}(2 m), y_{k, i}(2 m+1)\right] \\
& =\sum_{\ell=1}^{L}\left(g_{k, i}^{\ell}\right)^{*} \sum_{c=1}^{L}\left[v_{\ell, c}^{1}, v_{\ell, c}^{2}\right] \mathbf{C}\left(\mathbf{r}^{c}(m)\right)+\left[\mu_{k, i}(2 m), \mu_{k, i}(2 m+1)\right], \\
& =\sum_{\ell=1}^{L}\left(g_{k}^{\ell}\right)^{*} \sum_{c=1}^{L}\left[v_{\ell, c}^{1}, v_{\ell, c}^{2}\right]\left[\begin{array}{cc}
r^{c}(2 m) & r^{c}(2 m+1) \\
-r^{c}(2 m+1)^{*} & r^{c}(2 m)^{*}
\end{array}\right]+\left[\mu_{k, i}(2 m), \mu_{k, i}(2 m+1)\right], \\
& =\underbrace{\sum_{\ell=1}^{L} \sum_{c=1}^{L}\left[\left(g_{k, i}^{\ell}\right)^{*} v_{\ell, c}^{1} f_{k}^{c},\left(g_{k, i}^{\ell}\right)^{*} v_{\ell, c}^{2} f_{k}^{c *}\left[\begin{array}{cc}
s_{k}(2 m) & s_{k}(2 m+1) \\
-s_{k}(2 m+1)^{*} & s_{k}(2 m)^{*}
\end{array}\right]\right.}_{\text {desired signal }} \\
& +\underbrace{\sum_{\ell=1}^{L} \sum_{c=1}^{L} \sum_{j \neq k}\left[\left(g_{k, i}^{\ell}\right)^{*} v_{\ell, c}^{1} f_{j}^{c},\left(g_{k, i}^{\ell}\right)^{*} v_{\ell, c}^{2} f_{j}^{c *}\right]\left[\begin{array}{cc}
s_{j}(2 m) & s_{j}(2 m+1) \\
-s_{j}(2 m+1)^{*} & s_{j}(2 m)^{*}
\end{array}\right]}_{\text {interference signal }} \\
& +\underbrace{\sum_{\ell=1}^{L} \sum_{c=1}^{L}\left[\left(g_{k, i}^{\ell}\right)^{*} v_{\ell, c}^{1},\left(g_{k, i}^{\ell}\right)^{*} v_{\ell, c}^{2}\right]\left[\begin{array}{cc}
n^{\ell}(2 m) & n^{\ell}(2 m+1) \\
-n^{\ell}(2 m+1)^{*} & n^{\ell}(2 m)^{*}
\end{array}\right]+\left[\mu_{k, i}(2 m), \mu_{k, i}(2 m+1)\right]}_{\text {noise }} .
\end{aligned}
$$


By using a similar approach in identifying the signal-to-noise ratio (SNR) for the Alamouti coded signal in the point-to-point communication [41, Chapter 3.3.2], we can derive the SINR expression from $(20)$ by treating interference as white noise. Specifically, letting $\boldsymbol{w}_{1}=\operatorname{vec}\left(\boldsymbol{V}_{1}\right)$ and $\boldsymbol{w}_{2}=\operatorname{vec}\left(\boldsymbol{V}_{2}\right)$, the SINR at user- $(k, i)$ can be written as

$$
\frac{\boldsymbol{w}_{1}^{H} \boldsymbol{A}_{k, i} \boldsymbol{w}_{1}+\boldsymbol{w}_{2}^{H} \overline{\boldsymbol{A}}_{k, i} \boldsymbol{w}_{2}}{\boldsymbol{w}_{1}^{H} \boldsymbol{C}_{k, i} \boldsymbol{w}_{1}+\boldsymbol{w}_{2}^{H} \overline{\boldsymbol{C}}_{k, i} \boldsymbol{w}_{2}+1},
$$

where $\boldsymbol{A}_{k, i}, \overline{\boldsymbol{A}}_{k, i}, \boldsymbol{C}_{k, i}$ and $\overline{\boldsymbol{C}}_{k, i}$ are defined in (9), (22), (10) and (23). More details of the BFA AF detecting process can be found in the companion technical report [31]. Similarly, we can obtain $\overline{\boldsymbol{D}}_{\ell}$ and $\overline{\boldsymbol{G}}_{u}$ in (24) and (25).

\section{B. The Beamformed Alamouti AF Scheme for the distributed relay}

The BFA AF scheme for the distributed relay is similar to that for the MIMO case, and we defer the details of the derivations to the the companion technical report [31]. Note that for distributed relay network, we re-define $\boldsymbol{w}_{1}=\operatorname{Diag}\left(\boldsymbol{V}_{1}\right)$ and $\boldsymbol{w}_{2}=\operatorname{Diag}\left(\boldsymbol{V}_{2}\right)$, which is different from that in MIMO relay network. Therefore, the design problem for both the MIMO relay and distributed relay can be written as a two-variable fractional QCQP problem (R2BF) in Table II

\section{The SDR Technique for (R2BF)}

Problem (R2BF) is a fractional QCQP with two variables. It subsumes Problem (R1BF) when $\bar{A}=\mathbf{0}, \bar{C}=\mathbf{0}$ and $\bar{G}=\mathbf{0}$, and thus it is NP-hard. By applying SDR, we have the new AF design problem shown in Table II] where (R2SDR) serves as a convex relaxation to (R2BF). Define

$$
\theta\left(\boldsymbol{W}_{1}, \boldsymbol{W}_{2}\right)=\min _{\substack{i=1, \ldots, m_{k} \\ k=1, \ldots, G}} \frac{\boldsymbol{A}_{k, i} \bullet \boldsymbol{W}_{1}+\overline{\boldsymbol{A}}_{k, i} \bullet \boldsymbol{W}_{2}}{\boldsymbol{C}_{k, i} \bullet \boldsymbol{W}_{1}+\overline{\boldsymbol{C}}_{k, i} \bullet \boldsymbol{W}_{2}+1} .
$$

Clearly, we have

$$
\theta\left(\boldsymbol{W}_{1}^{\star}, \boldsymbol{W}_{2}^{\star}\right) \geq \theta\left(\boldsymbol{w}_{1}^{\star} \boldsymbol{w}_{1}^{\star H}, \boldsymbol{w}_{2}^{\star} \boldsymbol{w}_{2}^{\star H}\right),
$$

and equality holds whenever Problem (R2SDR) returns rank-one solutions. If $\boldsymbol{W}_{1}^{\star}$ or $\boldsymbol{W}_{2}^{\star}$ is non-rank-one, the Gaussian randomization algorithm in Algorithm 1 is applied to generate a sub-optimal solution $\left(\hat{\boldsymbol{w}}_{1}, \hat{\boldsymbol{w}}_{2}\right)$ to (R2BF) with $\theta\left(\boldsymbol{w}_{1}^{\star} \boldsymbol{w}_{1}^{\star H}, \boldsymbol{w}_{2}^{\star} \boldsymbol{w}_{2}^{\star H}\right) \geq \theta\left(\widehat{\boldsymbol{w}}_{1} \widehat{\boldsymbol{w}}_{1}^{H}, \widehat{\boldsymbol{w}}_{2} \widehat{\boldsymbol{w}}_{2}^{H}\right)$. To the best of our knowledge, the approximation quality of the solution $\left(\hat{\boldsymbol{w}}_{1}, \hat{\boldsymbol{w}}_{2}\right)$ has not been addressed in the literature before. In the next sub-section, we shall establish the first provable bound on the approximation quality of $\left(\hat{\boldsymbol{w}}_{1}, \hat{\boldsymbol{w}}_{2}\right)$. 
TABLE II

Summary of the Design Problem for BF Alamouti AF Schemes

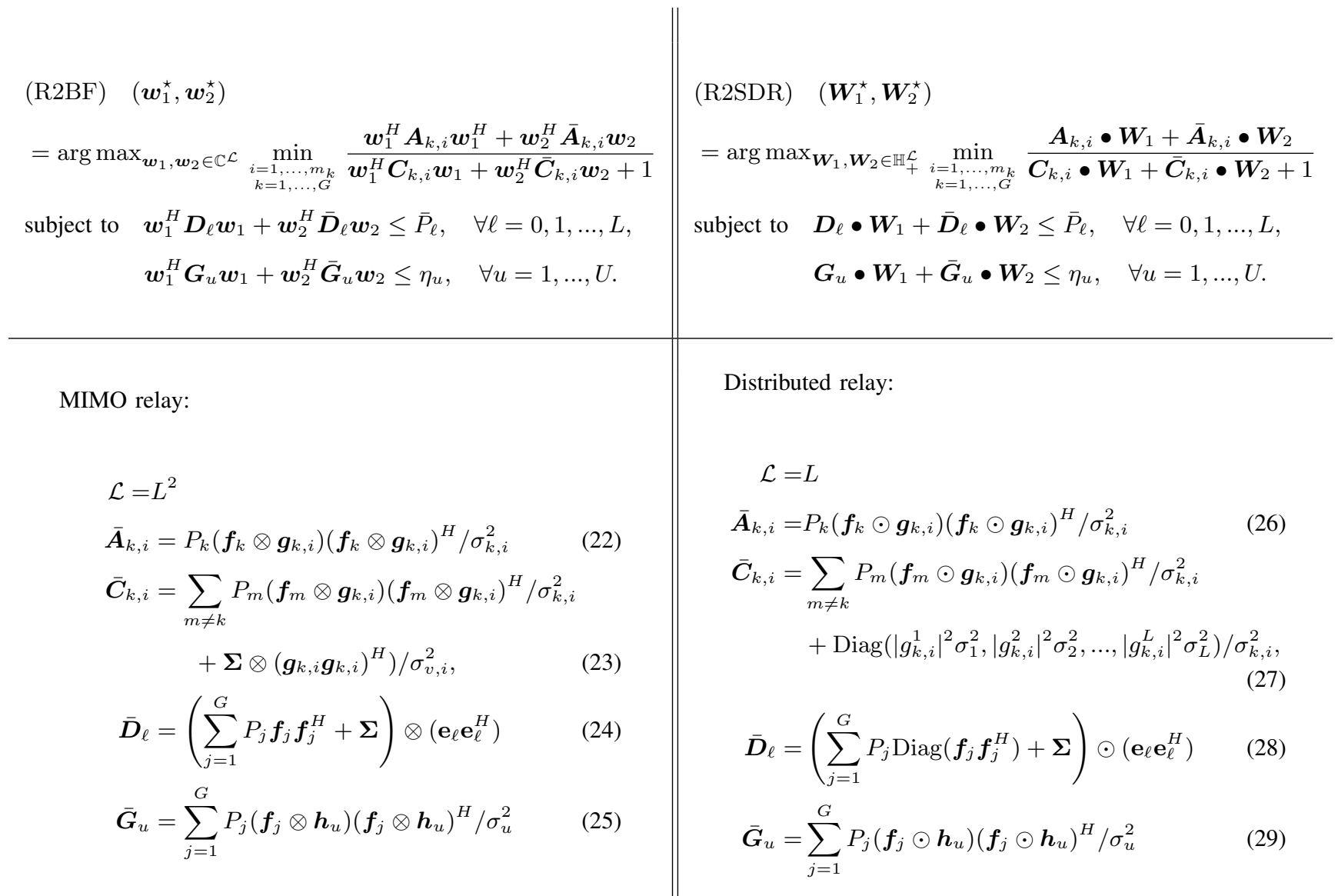

\section{SDR Approximation Bounds for BF Alamouti AF}

In this sub-section, we turn our attention to the performance analysis of the proposed BFA AF scheme. Specifically, we proceed by answering the following three questions.

1). What is the relationship between (R2SDR) and (R1SDR)?

2). When is (R2SDR) equivalent to $(\mathrm{R} 2 \mathrm{BF})$ ?

$3)$. What is the approximation quality of (R2SDR)?

Our recent works [22], [23] show that for the multigroup multicast network without relays, the design problems for BF AF and BFA AF have the same SDR, and the BFA AF scheme admits a rank-two approximate solution. However, the design problem for relay networks gives rise to two different SDRs, namely, (R1SDR) and (R2SDR). To answer Problem 1), it is easy to see 


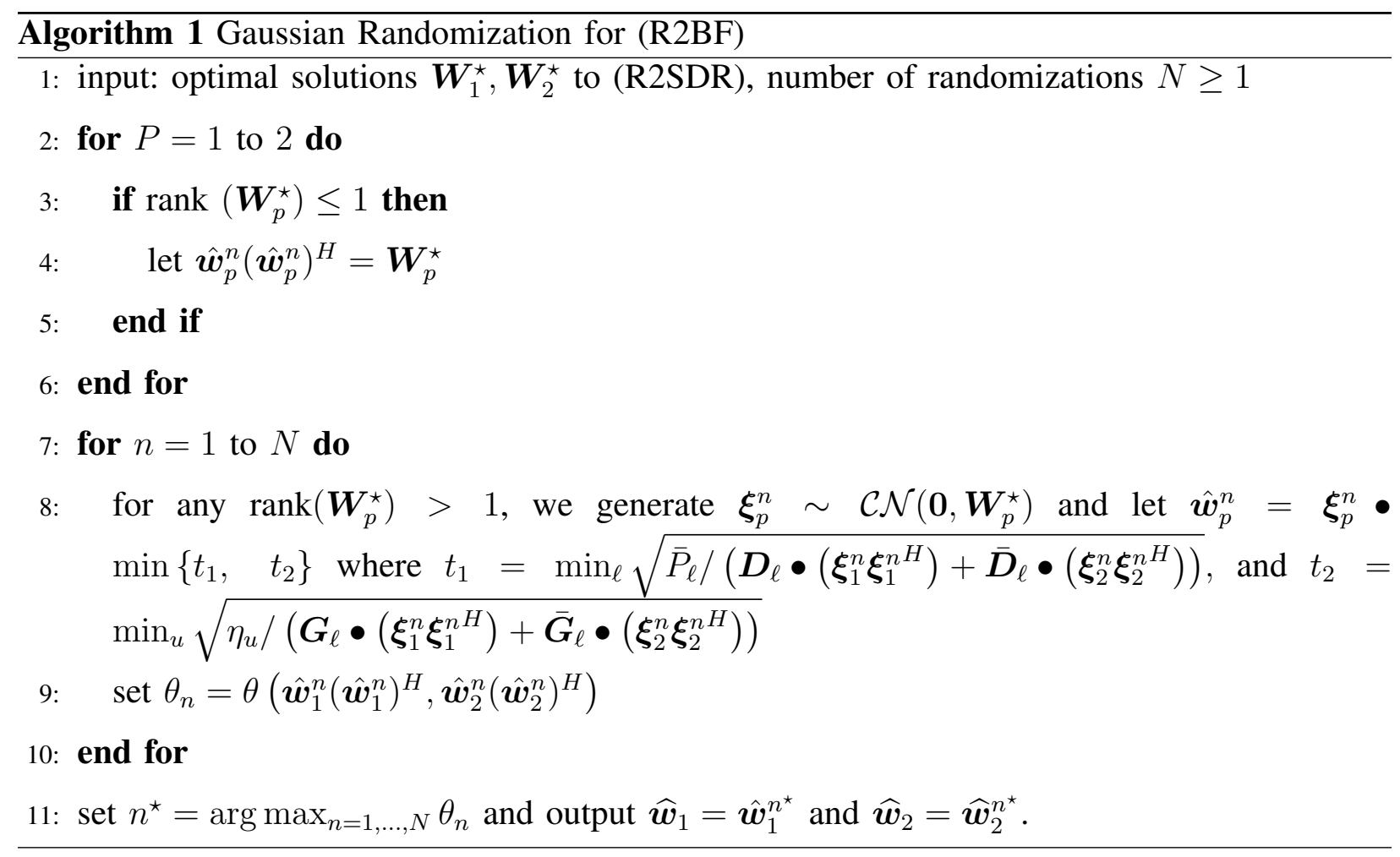

that

Proposition 2 The optimal value of (R2SDR) is always no worse than that of (RISDR).

The proof comes from the fact that $(\widehat{\boldsymbol{W}}, \mathbf{0})$ must be a feasible solution to (R2SDR), given that $\widehat{W}$ is feasible to (R1SDR). Intuitively, the gain also comes from the employment of one more DoF in the transmit structure.

To address Problem 2) and 3), we have the following theorem:

Theorem 1 Consider Problem (R2SDR). Let $M \geq 1$ be the total number of users in the relay network and $J$ denote the total number of constraints in $(R 1 B F)$, i.e., $J=L+U+1$ and $J \geq 1$. Let $\boldsymbol{W}_{1}^{\star}$ and $\boldsymbol{W}_{2}^{\star}$ be the optimal solutions to Problem (R2SDR) and we consider the cases of both $\boldsymbol{W}_{1}^{\star} \neq \mathbf{0}$ and $\boldsymbol{W}_{2}^{\star} \neq 0 . !^{3}$ Then, we have the following results:

a). Problem $(R 2 S D R)$ can produce an optimal solution to Problem $(R 2 B F)$ when $M+J \leq 5$.

\footnotetext{
${ }^{3}$ It is a trivial case if $\boldsymbol{W}_{1}^{\star}=\boldsymbol{W}_{2}^{\star}=\mathbf{0}$. If either $\boldsymbol{W}_{1}^{\star}=\mathbf{0}$ or $\boldsymbol{W}_{2}^{\star}=\mathbf{0}$, it reduces to the rank-one case and (R2SDR) is optimal for (R2BF) when $M+J \leq 4$.
} 
b). When $M+J>5$ and $\operatorname{rank}\left(\boldsymbol{W}_{p}^{\star}\right)>1$ for some $p=1,2$ in $(R 2 S D R)$, let $\widehat{\boldsymbol{w}}_{1}, \widehat{\boldsymbol{w}}_{2}$ be the solutions returned by Algorithm 1 and $J=L+U+1$ be the total number of power constraints. Then, with probability at least $1-(7 / 8)^{N}$, we have

$$
\theta\left(\widehat{\boldsymbol{w}}_{1} \widehat{\boldsymbol{w}}_{1}^{H}, \widehat{\boldsymbol{w}}_{2} \widehat{\boldsymbol{w}}_{2}^{H}\right) \geq c \cdot \theta\left(\boldsymbol{w}_{1}^{\star} \boldsymbol{w}_{1}^{\star H}, \boldsymbol{w}_{2}^{\star} \boldsymbol{w}_{2}^{\star H}\right),
$$

where

$$
\begin{gathered}
c=\frac{\max \left\{\frac{\omega}{7 \sqrt{M}}, \frac{1}{8 M}\right\}}{2 \log (16 J)+1}, \\
\omega=\min _{k, i}\left\{\frac{\min \left\{\boldsymbol{A}_{k, i} \bullet \boldsymbol{W}_{1}^{\star}, \overline{\boldsymbol{A}}_{k, i} \bullet \boldsymbol{W}_{2}^{\star}\right\}}{\boldsymbol{A}_{k, i} \bullet \boldsymbol{W}_{1}^{\star}+\overline{\boldsymbol{A}}_{k, i} \bullet \boldsymbol{W}_{2}^{\star}}\right\}, \text { and } N \text { is the number of randomizations. }
\end{gathered}
$$

The proof of Theorem [1a) is similar to that for Theorem 1 in [10]; see details in Appendix A in [10] and [42, Theorem 5.1]. We relegate the proof of Theorem 1 $1 \mathrm{~b}$ ) in Appendix $\mathrm{A}$ at the end of this paper. The significance of Theorem 1 is twofold. First, it shows that (R2SDR) is optimal for BFA AF when $M+J \leq 5$. Then, in conjunction with Proposition 2 , the proposed method must be better than BF AF in the case of $M+J \leq 5$. Second, if (R2SDR) does not have rank-one solutions, Theorem 1 specifies the quality of the SDR solution returned by the Gaussian randomization algorithm-i.e., on the order of $\frac{1}{\sqrt{M} \log J}$ if $\frac{\omega}{7 \sqrt{M}} \geq \frac{1}{8 M}$; on the order of $\frac{1}{M \log J}$ if $\frac{\omega}{7 \sqrt{M}}<\frac{1}{8 M}$. If we fix the number of power constraints $J$ and increase the number of users in the system, then we see that $\Omega(1 / \sqrt{M})$ scales better than $\Omega(1 / M)$. Moreover, we see from (32) that the approximation quality is lower bounded by $\Omega(1 / M)$. Hence, in conjunction with Proposition 2, the performance of BFA AF is provably no worse than that of BF AF. Remark 2: One may observe that (R2BF) can be written in the form of (R1BF) by letting $\boldsymbol{w}=\left[\boldsymbol{w}_{1} ; \boldsymbol{w}_{2}\right]$ and $\tilde{\boldsymbol{A}}_{k, i}=\left[\boldsymbol{A}_{k, i}, \mathbf{0} ; \mathbf{0}, \overline{\boldsymbol{A}}_{k, i}\right]$. Thus, it immediately admits the inferior bound in Proposition 1. Moreover, if $\overline{\boldsymbol{A}}_{k, i}=\mathbf{0}$, the approximation bound in Theorem 1 reduces to that in Proposition 1 . Thus Theorem 1 can be seen as a generalization of Proposition 1 from one-variable fractional QCQPs to two-variable fractional QCQPs.

Remark 3: If we let $\overline{\boldsymbol{A}}_{k, i}=\boldsymbol{A}_{k, i}$ and $\overline{\boldsymbol{C}}_{k, i}=\boldsymbol{C}_{k, i}$, Problem (R2BF) will be reduced to Problem (R1BF) by replacing the rank-one variable $\boldsymbol{w}$ to a rank-two matrix $B$. We have studied the latter in [22] by considering the multigroup multicast transmission in a network without relays, where the SDR rank-two approximation bounds are provided based on Problem (R1SDR). Hence, our results in Theorem 1 also generalize the approximation bounds in [22, Theorem 1]. 


\section{Special Cases with Deterministic Approximation Bounds}

In this section, by considering some special cases of (R2BF), we will demonstrate how the two DoFs offered by the BFA AF scheme can lead to improved performance over the BF AF scheme. In particular, we consider special cases in both distributed relay network and MIMO relay network, where by exploiting the special structures of the problems, we can obtain various strengthenings of Theorem 1 .

\section{A. Multicasting for Distributed Relay Networks}

For the distributed relay case, by observing the structure of $\boldsymbol{A}_{k, i}$ and $\overline{\boldsymbol{A}}_{k, i}$, we can define $\phi_{\ell}=\arg \left(f_{k}^{\ell}\right)$ and $\mathbf{e}=\left[e^{j 2 \phi_{1}}, \ldots, e^{j 2 \phi_{\ell}}, \ldots, e^{j 2 \phi_{L}}\right]^{T}$ with $j=\sqrt{-1}$ such that

$$
\boldsymbol{w}_{2}^{H} \overline{\boldsymbol{A}}_{k, i} \boldsymbol{w}_{2}=\left(\boldsymbol{w}_{2} \odot \mathbf{e}\right)^{H} \boldsymbol{A}_{k, i}\left(\boldsymbol{w}_{2} \odot \mathbf{e}\right)
$$

and

$$
\boldsymbol{w}_{2}^{H} \overline{\boldsymbol{G}}_{u} \boldsymbol{w}_{2}=\left(\boldsymbol{w}_{2} \odot \mathbf{e}\right)^{H} \boldsymbol{G}_{u}\left(\boldsymbol{w}_{2} \odot \mathbf{e}\right) .
$$

Then, letting $\overline{\boldsymbol{w}}_{2}=\boldsymbol{w}_{2} \odot \mathbf{e}$, we actually have

$$
\overline{\boldsymbol{A}}_{k, i} \bullet \boldsymbol{w}_{2} \boldsymbol{w}_{2}^{H}=\boldsymbol{A}_{k, i} \bullet \overline{\boldsymbol{w}}_{2} \overline{\boldsymbol{w}}_{2}^{H}
$$

and

$$
\overline{\boldsymbol{G}}_{u} \bullet \boldsymbol{w}_{2} \boldsymbol{w}_{2}^{H}=\boldsymbol{G}_{u} \bullet \overline{\boldsymbol{w}}_{2} \overline{\boldsymbol{w}}_{2}^{H} .
$$

On the other hand, we also have

$$
\overline{\boldsymbol{C}}_{k, i} \bullet \overline{\boldsymbol{w}}_{2} \overline{\boldsymbol{w}}_{2}^{H}=\boldsymbol{C}_{k, i} \bullet \boldsymbol{w}_{2} \boldsymbol{w}_{2}^{H},
$$

since $C_{k, i}$ must be a diagonal matrix in the target relay network. Moreover, for distributed relays, we have $\overline{\boldsymbol{D}}_{\ell}=\boldsymbol{D}_{\ell}, \quad \forall \ell=0, \ldots, L$, and $\boldsymbol{D}_{\ell}$ s are also diagonal. Therefore

$$
\boldsymbol{D}_{\ell} \bullet \overline{\boldsymbol{w}}_{2} \overline{\boldsymbol{w}}_{2}^{H}=\boldsymbol{D}_{\ell} \bullet \boldsymbol{w}_{2} \boldsymbol{w}_{2}^{H}, \quad \forall \ell=1, \ldots, L,
$$

for the diagonal matrix $\boldsymbol{D}_{\ell}$. Thus, Problem (R2BF) can be rewritten as

$$
\begin{array}{cl}
\max _{\boldsymbol{w}_{1}, \boldsymbol{w}_{2} \in \mathbb{C}^{\mathcal{L}}} \min _{\substack{i=1, \ldots, m_{k} \\
k=1, \ldots, G}} \frac{\boldsymbol{w}_{1}^{H} \boldsymbol{A}_{k, i} \boldsymbol{w}_{1}+\overline{\boldsymbol{w}}_{2}^{H} \boldsymbol{A}_{k, i} \overline{\boldsymbol{w}}_{2}}{\boldsymbol{w}_{1}^{H} \boldsymbol{C}_{k, i} \boldsymbol{w}_{1}+\overline{\boldsymbol{w}}_{2}^{H} \boldsymbol{C}_{k, i} \overline{\boldsymbol{w}}_{2}+1} \\
\text { subject to } & \boldsymbol{w}_{1}^{H} \boldsymbol{D}_{\ell} \boldsymbol{w}_{1}+\overline{\boldsymbol{w}}_{2}^{H} \boldsymbol{D}_{\ell} \overline{\boldsymbol{w}}_{2} \leq \bar{P}_{\ell}, \quad \ell=0,1, \ldots, L, \\
& \boldsymbol{w}_{1}^{H} \boldsymbol{G}_{u} \boldsymbol{w}_{1}+\overline{\boldsymbol{w}}_{2}^{H} \boldsymbol{G}_{u} \overline{\boldsymbol{w}}_{2} \leq \eta_{u}, \quad u=0,1, \ldots, U,
\end{array}
$$


where we have $\mathcal{L}=L$. Clearly, the resulting SDR of (39) has the same optimal value as that of (R1SDR). Moreover, it is easy to see that we have $\omega=1 / 2$ here since $\boldsymbol{A}_{k, i}=\overline{\boldsymbol{A}}_{k, i}$ for all $k, i$. Note that herein we have $k=1$ for multicasting. Thus, we may conclude

Proposition 3 For distributed relay multicasting with generalized power constraints, Problem $(R 2 B F)$ can be rewritten as Problem (39), and therefore the approximation bound

$$
\theta\left(\widehat{\boldsymbol{w}}_{1} \widehat{\boldsymbol{w}}_{1}^{H}, \widehat{\boldsymbol{w}}_{2} \widehat{\boldsymbol{w}}_{2}^{H}\right)=\Omega\left(\frac{1}{\sqrt{M} \log J}\right) \theta\left(\boldsymbol{w}_{1}^{\star} \boldsymbol{w}_{1}^{\star H}, \boldsymbol{w}_{2}^{\star} \boldsymbol{w}_{2}^{\star H}\right)
$$

holds with probability at least $1-(7 / 8)^{N}$.

This result can also be obtained by applying the approximation bound results in [22] to (R1SDR). It is worth mentioning that prior to this proposition, the authors in [24] have provided a similar result by introducing a phase rotation at the $\mathrm{AF}$ process under a total power constraint. Nevertheless, our derivation here reveals how the two DoFs lead to a gain in performance.

\section{B. Multicasting for MIMO Relay Networks}

We also have some interesting results for the MIMO relay multicasting network. It shows that in this case, the AF scheme possesses some special structure, which leads to an approximation bound of $\Omega\left(\frac{1}{\sqrt{M} \log J}\right)$. We summarize the result in Proposition 4 .

Proposition 4 For MIMO relay multicasting with generalized power constraints, Problem $(R 2 B F)$ can be rewritten as Problem (39) with proper parameter definitions for MIMO relays, and therefore the approximation bound

$$
\theta\left(\widehat{\boldsymbol{w}}_{1} \widehat{\boldsymbol{w}}_{1}^{H}, \widehat{\boldsymbol{w}}_{2} \widehat{\boldsymbol{w}}_{2}^{H}\right)=\Omega\left(\frac{1}{\sqrt{M} \log J}\right) \theta\left(\boldsymbol{w}_{1}^{\star} \boldsymbol{w}_{1}^{\star H}, \boldsymbol{w}_{2}^{\star} \boldsymbol{w}_{2}^{\star H}\right)
$$

holds with probability at least $1-(7 / 8)^{N}$.

The proof is similar to that in Proposition 3, except that we now define $\phi_{\ell}=\arg \left(f_{k}^{\ell}\right)$ and $\mathbf{e}=\left[e^{j 2 \phi_{1}}, \ldots, e^{j 2 \phi_{\ell}}, \ldots, e^{j 2 \phi_{L}}\right]^{T} \otimes \mathbf{1}$ such that equations (33)-(38) hold for the MIMO relay case. This proposition reveals that for MIMO relay multicasting, the SDR approximation bound is on the order of $1 /(\sqrt{M} \log J)$, which is definitely better than that for the BF AF counterpart. It is worth noting that the optimal values for (R1SDR) and (R2SDR) are the same, and thus the SINR performance is improved due to the better approximation quality. This demonstrates how the proposed BFA AF scheme benefits the system performance. 


\section{Numerical Simulations}

In this section, we provide numerical simulations to compare the performance of different AF schemes and demonstrate the superiority of the proposed BFA AF scheme. We show the numerical results for the distributed relay network in this section and defer the results for the MIMO relay network and the comparison with the feasible point pursuit (FPP) algorithm [28], [29] in the the companion technical report [31]. We assume w.l.o.g. that each multicast group has an equal number of users (i.e., $m_{k}=M / G$ for $k=1, \ldots, G$ ). The channels $\boldsymbol{f}_{k}, \boldsymbol{g}_{k, i}$, where $k=1, \ldots, G$ and $i=1, \ldots, m_{k}$, are identical independently distributed (i.i.d.) according to $\mathcal{C N}(\mathbf{0}, \boldsymbol{I})$. The transmitted signal at each transmitter is with power $0 \mathrm{~dB}$ (i.e., $P_{j}=0 \mathrm{~dB}$ for $j=1, \ldots, G)$. Each single-antenna relay has the same noise power (i.e., $\sigma_{\ell}^{2}=\sigma_{\text {ant }}^{2}$, where $\ell=1, \ldots, L)$, and all users have the same noise power (i.e., $\sigma_{k, i}^{2}=\sigma_{\text {user }}^{2}$ for $k=1, \ldots, G$ and $\left.i=1, \ldots, m_{k}\right)$. We assume that $\sigma_{\text {ant }}^{2}>0$ and $\sigma_{\text {user }}^{2}>0$. The total power threshold for all the relays is $\bar{P}_{0}$; the power threshold at $\ell$ th relay is $\bar{P}_{\ell}$, where $\ell=1, \ldots, L$. For each AF scheme, 100 channel realizations were averaged to get the plots, and the number of randomizations for generating BF AF weights and BFA weights is 1,000 .

\section{A. Worst User's SINR versus Total Power Threshold}

In this simulation, we vary the total power budget at relays to see the worst user's SINR performance in different relay networks. For ease of exposition, we consider the scenario where only the total power constraint is present. In Figure 4, we assume that there are $L=8$ singleantenna relays and $G=2$ multicast groups with a total of $M=16$ users. For both cases, we set $\sigma_{\text {ant }}^{2}=\sigma_{\text {user }}^{2}=0.25$. It shows that the objective value (obj.) of (R1SDR) and (R2SDR) serve as an upper bound for the SDR-based BF AF scheme and the SDR-based BFA AF scheme, respectively. Moreover, based on randomization, the BFA AF scheme shows a significant SINR improvement over the BF AF scheme in all the power regions.

\section{B. Worst User's SINR versus Number of Users}

In this simulation, we show how the worst user's SINR scales with the number of users served in the relay system. We consider the problem formulation with one total power constraint present. In Figure 5, we have $L=8, G=2, \sigma_{\text {ant }}^{2}=\sigma_{\text {user }}^{2}=0.25$ and the total power threshold at relays is set to be $\bar{P}_{0}=10 \mathrm{~dB}$. From the figure, we see that (R2SDR) serves as an upper bound of 


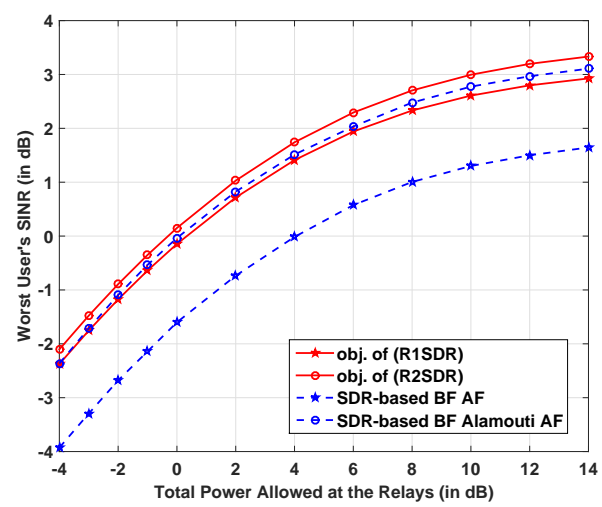

Fig. 4. Worst user's SINR versus total power threshold at the distributed relay: $L=8, G=2, M=12, \sigma_{\text {ant }}^{2}=\sigma_{\text {user }}^{2}=0.25$.

(R1SDR), which is consistent with Propositions 2 and 3. Moreover, it shows that BFA AF can own better SINR performance than the BF AF scheme for all the values of $M$, which verifies the results in Proposition 1 and Theorem 1 .

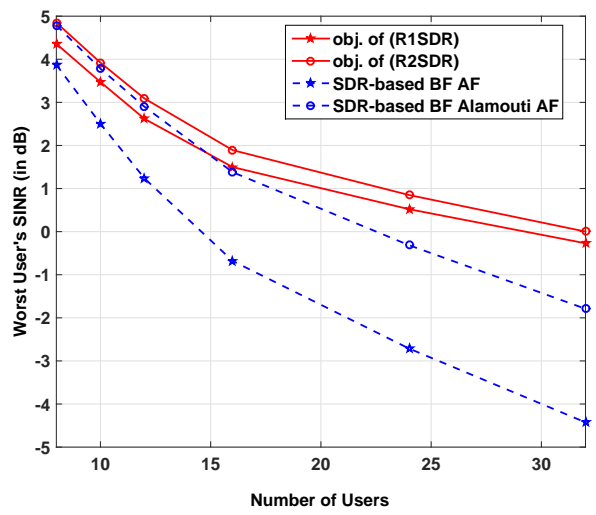

Fig. 5. Worst user's SINR versus number of users in the distributed relay system: $L=8, G=2, \bar{P}_{0}=10 \mathrm{~dB}, \sigma_{\text {ant }}^{2}=\sigma_{\text {user }}^{2}=$ 0.25 .

\section{Worst User's SINR versus Number of Per-relay Power Constraints}

In this simulation, we consider the scenario where both the total power constraint and perantenna power constraints are present and the primal users are absent. Our purpose is to see how the worst user's SINR scales with the number of per-relay power constraints. Specifically, Figure 6 shows the distributed relay case with $L=8, G=2, M=16$, where the total power threshold is $\bar{P}_{0}=7 \mathrm{~dB}$ and per-relay power threshold is also $\bar{P}_{\ell}=-5 \mathrm{~dB}$. We set $\sigma_{\text {ant }}^{2}=\sigma_{\text {user }}^{2}=0.25$ 
and vary the number of per-relay power constraints from 0 to $L$ to compare SINR performances of different AF schemes. It shows that the BF Alamouti AF scheme outperforms the BF AF scheme. As the number of per-relay power constraints increases, the SINRs diverge from their SDR upper bounds, and both $\mathrm{BF} \mathrm{AF}$ and $\mathrm{BF}$ Alamouti AF exhibit the same scaling with $L$, which is consistent with the approximation bounds in terms of $J$ in Proposition 1 and Theorem 1.

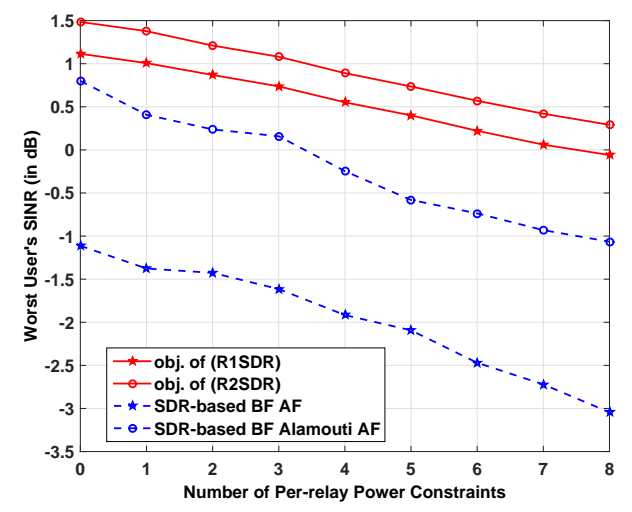

Fig. 6. Worst user's rate achieved by different AF schemes versus number of per-antenna power constraints in the distributed relay network: $L=8, G=2, M=16, \bar{P}_{0}=7 \mathrm{~dB}, \bar{P}_{\ell}=-5 \mathrm{~dB}$ for $\ell=1, \ldots, L, \sigma_{\text {ant }}^{2}=\sigma_{\text {user }}^{2}=0.25$.

\section{Worst User's SINR versus Number of Primal Users}

Similar to previous simulations, here we show the worst user's SINR scaling with the number of primal users. To set up the problem, we consider the scenario where the total power constraint and the primal users' interference constraints are present. We assume that $L=8, G=2$ and $M=12$ and set $\sigma_{\text {ant }}^{2}=\sigma_{\text {user }}^{2}=0.25$, the total power budget $\bar{P}_{0}=10 \mathrm{~dB}$, and the noise power at all primal users to be $\sigma_{\mathrm{u}}^{2}=0.25$. Moreover, we assume that the primal users are subject to an interference power threshold equaling to $b_{u}=3 \mathrm{~dB}$. We then increase the number of primal users to see the SINR scaling in Figure 7. It shows that as the primal users increases, both the BF AF scheme and BFA AF scheme diverge from their SDR bounds and BFA AF shows a significant improvement over BF AF. These results further validate Proposition 1 and Theorem 1 in terms of the scaling of $J$. 


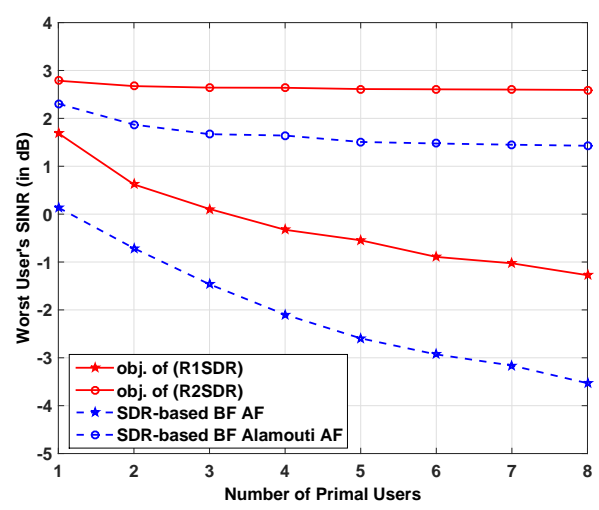

Fig. 7. Worst user's SINR versus number of primal users in the distributed relay CR network: $L=8, G=2, M=12$, $\bar{P}_{0}=10 \mathrm{~dB}, b_{u}=3 \mathrm{~dB}$ for $u=1, \ldots, U, \sigma_{\text {ant }}^{2}=\sigma_{\text {user }}^{2}=0.25$ and $\sigma_{\mathrm{u}}^{2}=0.25$.

\section{E. Simulation Results for Multicasting Relay Networks}

In this sub-section, we provide numerical results for the special cases of multicasting. In both Figures 8 and 9 , we consider the problem formulation with the total power constraint and one primal user's temperature constraint and show the worst user's SINR scaling with the number of users. Specifically, in Figure 8, we set $L=8, \bar{P}_{0}=10 \mathrm{~dB}$ and $\sigma_{\text {ant }}^{2}=\sigma_{\text {user }}^{2}=0.25$. The simulation results show that the obj. of (R1SDR) and (R2SDR) coincide with each other, and the curve of BFA AF scales better than that for BF AF, which is consistent with results in Proposition 3. In Figure 9, we have $L=4, \bar{P}_{0}=10 \mathrm{~dB}$ and $\sigma_{\text {ant }}^{2}=\sigma_{\text {user }}^{2}=0.25$. It shows that the obj. of (R2SDR) equal to that of (R1SDR). Moreover, BFA AF exhibits better scaling than $\mathrm{BF}$ AF as the number of users increases. This verifies results in Proposition 4.

\section{F. Actual Bit Error Rate (BER) Performance}

To further demonstrate the efficacy of the proposed AF scheme, we study the actual coded bit error rate (BER) performance of the scenario setting in Figure 4. Specifically, for each time slot, we simulate the actual AF process by generating $s_{j}(t), n^{\ell}(t)$ according to the SISO model in (4) and (20) and detecting and decoding $s_{j}(t)$ at each receivers. The resulting BERs are shown in Figures 10. To simulate the SDR bound in the BER plots, we assume that there exists an SISO channel whose SINR is equal to $\gamma\left(\boldsymbol{W}^{\star}\right)$ or $\theta\left(\boldsymbol{W}_{1}^{\star}, \boldsymbol{W}_{2}^{\star}\right)$. In our simulations, we adopt a gray-coded QPSK modulation scheme and a rate- $1 / 3$ turbo code in [43] with a codelength of 


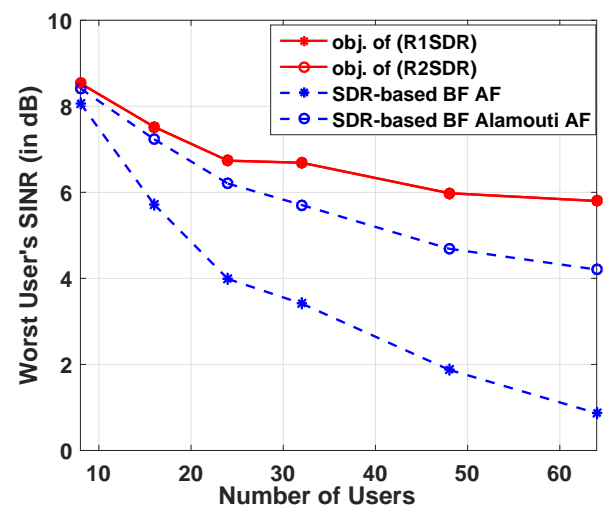

Fig. 8. Worst user's SINR versus the number of users in the distributed relay multicast network: $L=8, G=1, M=16$, $\bar{P}_{0}=10 \mathrm{~dB}, b_{u}=3 \mathrm{~dB}, \sigma_{\text {ant }}^{2}=\sigma_{\text {user }}^{2}=0.25$ and $\sigma_{\mathrm{u}}^{2}=0.25$.

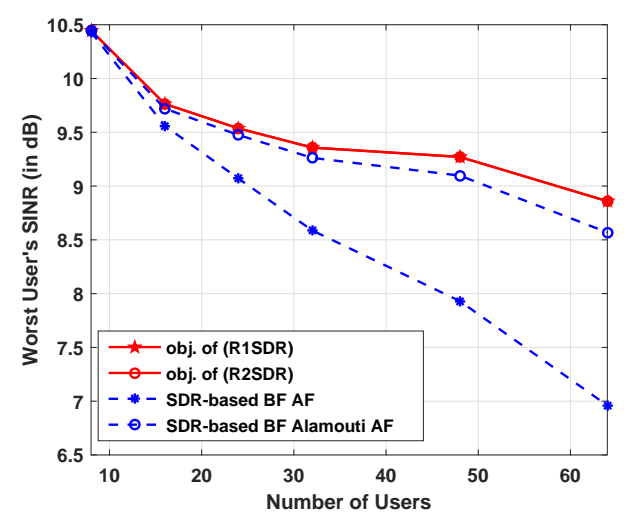

Fig. 9. Worst user's SINR versus the number of users in the MIMO relay multicast network: $L=4, G=1, M=16$, $\bar{P}_{0}=10 \mathrm{~dB}, b_{u}=3 \mathrm{~dB}, \sigma_{\text {ant }}^{2}=\sigma_{\text {user }}^{2}=0.25$ and $\sigma_{\mathrm{u}}^{2}=0.25$

2880 bits. We simulate 100 code blocks for each channel realization and thus the BER reliability level is $10 \mathrm{e}-4$. We see that the actual BER performance of the proposed BFA AF scheme indeed outperforms the BF-AF scheme at almost all power thresholds. The results are consistent with those SINR results in Figure 4 and show that BFA AF can achieve a good performance in real applications.

\section{CONCLUSIONS}

In this work, we have studied the AF design problem in both the MIMO relay network and the distributed relay network. We considered different power constraints and tried to maximize the worst user's SINR under these power constraints in the multigroup multicasting scenario. 


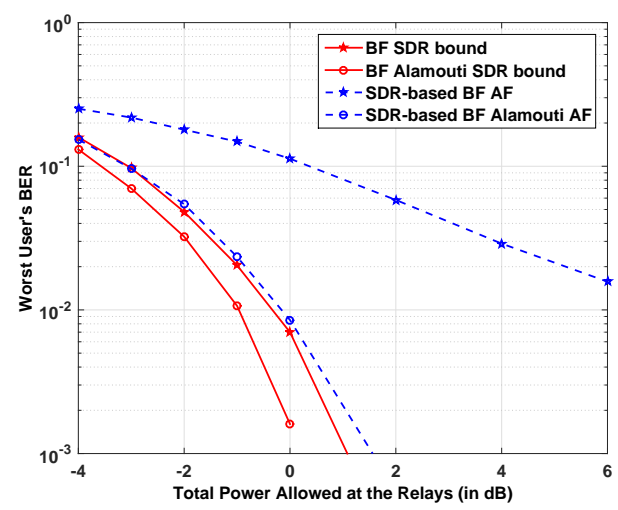

Fig. 10. Worst user's BER achieved by different AF schemes versus total power threshold in the distributed relay network: $L=8, G=2, M=12, \sigma_{\text {ant }}^{2}=\sigma_{\text {user }}^{2}=0.25$. A rate- $\frac{1}{3}$ turbo code with codelength 2880 is used.

A novel BFA AF scheme was proposed, for which the design problem is formulated as a twovariable fractional QCQP. Our main contributions lie in two aspects. First, we analyzed the new two-variable fractional SDR problem. The results show that by introducing 2-DoF in the AF structure, we can increase the optimal objective value of the SDR problem. Moreover, the quality the SDR solution also improves, and therefore system performance can be enhanced. Second, we showed in some special cases of relay multicasting that how the introduced one more DoF benefits the system performance and derived some interesting results, which can facilitate the system design. In summary, we demonstrate from both theoretical and numerically ways that the proposed BFA AF scheme can outperform the traditional BF AF scheme, especially in largescale systems. Our future work is the AF design under imperfect CSI scenarios and other types of relay cloud networks with limited link capacity.

\section{APPENDIX}

\section{A. Proof of Theorem 1}

In this appendix, we provide the proof of Theorem 1 in four steps. Specifically, we begin by considering a specific randomization time $n$ in Algorithm 1. W.l.o.g, we let $\boldsymbol{R}_{j}$ and $\overline{\boldsymbol{R}}_{j}$ denote the matrices associated with the power constraints, e.g., $\boldsymbol{R}_{j}=\boldsymbol{G}_{u}, \overline{\boldsymbol{R}}_{j}=\overline{\boldsymbol{G}}_{u}$ or $\boldsymbol{R}_{j}=\overline{\boldsymbol{R}}_{j}=\boldsymbol{D}_{\ell}$. Hence, herein $J$ is the total number of generalized power constraints. Letting $\boldsymbol{W}_{1}=\left(\boldsymbol{\xi}_{1}^{n}\right)\left(\boldsymbol{\xi}_{1}^{n}\right)^{H}$ and $\boldsymbol{W}_{2}=\left(\boldsymbol{\xi}_{2}^{n}\right)\left(\boldsymbol{\xi}_{2}^{n}\right)^{H}$, our purpose in Steps 1-3 is to prove that for any proper $\rho, v>0$, the 
probability

$$
\begin{aligned}
& \mathcal{P} \triangleq \operatorname{Pr}\left(\left\{\theta\left(\boldsymbol{W}_{1}, \boldsymbol{W}_{2}\right) \geq \rho \cdot \theta\left(\boldsymbol{W}_{1}^{\star}, \boldsymbol{W}_{2}^{\star}\right)\right\}\right. \\
& \wedge\left\{\boldsymbol{R}_{j} \bullet \boldsymbol{W}_{1}+\overline{\boldsymbol{R}}_{j} \bullet \boldsymbol{W}_{2} \leq v\left(\boldsymbol{R}_{j} \bullet \boldsymbol{W}_{1}^{\star}+\overline{\boldsymbol{R}}_{j} \bullet \boldsymbol{W}_{2}^{\star}\right),\right. \\
& \quad \forall j=1, \ldots, J\})
\end{aligned}
$$

is always positive. Based on this result, in Step 4, we could further identify the approximation bounds.

Step 1: To proceed the proof for $\mathcal{P}>0$, we define

$$
\theta_{k, i}\left(\boldsymbol{X}_{1}, \boldsymbol{X}_{2}\right)=\frac{\boldsymbol{A}_{k, i} \bullet \boldsymbol{X}_{1}+\overline{\boldsymbol{A}}_{k, i} \bullet \boldsymbol{X}_{2}}{\boldsymbol{C}_{k, i} \bullet \boldsymbol{X}_{1}+\overline{\boldsymbol{C}}_{k, i} \bullet \boldsymbol{X}_{2}+1}
$$

Then, let us consider the events

$$
\begin{aligned}
& \mathcal{E}_{k, i}=\left\{\theta_{k, i}\left(\boldsymbol{W}_{1}, \boldsymbol{W}_{2}\right) \leq \rho \cdot \theta_{k, i}\left(\boldsymbol{W}_{1}^{\star}, \boldsymbol{W}_{2}^{\star}\right)\right\} \\
& \mathcal{F}_{j}=\left\{\boldsymbol{R}_{j} \bullet \boldsymbol{W}_{1}+\overline{\boldsymbol{R}}_{j} \bullet \boldsymbol{W}_{2} \geq v\left(\boldsymbol{R}_{j} \bullet \boldsymbol{W}_{1}^{\star}+\overline{\boldsymbol{R}}_{j} \bullet \boldsymbol{W}_{2}^{\star}\right)\right\}
\end{aligned}
$$

and define $\mathcal{E}_{k, i}^{c}$ and $\mathcal{F}_{j}^{c}$ to be the complement of $\mathcal{E}_{k, i}$ and $\mathcal{F}_{j}$. It is easy to see that

$$
\mathcal{P} \geq 1-\operatorname{Pr}\left(\bigcup_{\substack{k=1, \ldots, G \\ i=1, \ldots, m_{k}}} \mathcal{E}_{k, i}\right)-\operatorname{Pr}\left(\bigcup_{j=1}^{J} \mathcal{F}_{j}\right) .
$$

Step 2: We intend to use the following Lemma 1 to bound $\operatorname{Pr}\left(\bigcup_{\substack{k=1, \ldots, G \\ i=1, \ldots, m_{k}}} \mathcal{E}_{k, i}\right)$.

Lemma 1 Given Hermitian positive semidefinite matrices $\boldsymbol{A}, \overline{\boldsymbol{A}}, \boldsymbol{C}, \overline{\boldsymbol{C}}$ with $\operatorname{rank}(\boldsymbol{A})=\operatorname{rank}(\overline{\boldsymbol{A}})=$ 1 , let $\boldsymbol{\xi} \sim \mathcal{C N}\left(\mathbf{0}, \boldsymbol{X}_{1}^{\star}\right), \boldsymbol{\eta} \sim \mathcal{C N}\left(\mathbf{0}, \mathbf{X}_{2}^{\star}\right)$ be independent random vectors. Assume that $\boldsymbol{A} \bullet \boldsymbol{X}_{1}^{\star}>0$ and $\overline{\boldsymbol{A}} \bullet \boldsymbol{X}_{2}^{\star}>0$. Then,

1). For any $\rho<1 / 2$, it holds that

$$
\begin{aligned}
& \operatorname{Pr}\left(\frac{\boldsymbol{\xi}^{H} \boldsymbol{A} \boldsymbol{\xi}+\boldsymbol{\eta}^{H} \overline{\boldsymbol{A}} \boldsymbol{\eta}}{\boldsymbol{\xi}^{H} \boldsymbol{C} \boldsymbol{\xi}+\boldsymbol{\eta}^{H} \overline{\boldsymbol{C}} \boldsymbol{\eta}+1} \leq \rho \frac{\boldsymbol{A} \bullet \boldsymbol{X}_{1}^{\star}+\overline{\boldsymbol{A}} \bullet \boldsymbol{X}_{2}^{\star}}{\boldsymbol{C} \bullet \boldsymbol{X}_{1}^{\star}+\overline{\boldsymbol{C}} \bullet \boldsymbol{X}_{2}^{\star}+1}\right) \\
& \leq \frac{4 \rho}{1-2 \rho}
\end{aligned}
$$

2). For for any $\rho<\omega / 2$ where $\omega=\frac{\min \left\{\boldsymbol{A} \bullet \boldsymbol{X}_{1}^{\star}, \overline{\boldsymbol{A}} \bullet \boldsymbol{X}_{2}^{\star}\right\}}{\boldsymbol{A} \bullet \boldsymbol{X}_{1}^{\star}+\overline{\boldsymbol{A}} \bullet \boldsymbol{X}_{2}^{\star}}$, it holds that

$$
\begin{aligned}
& \operatorname{Pr}\left(\frac{\boldsymbol{\xi}^{H} \boldsymbol{A} \boldsymbol{\xi}+\boldsymbol{\eta}^{H} \overline{\boldsymbol{A}} \boldsymbol{\eta}}{\boldsymbol{\xi}^{H} \boldsymbol{C} \boldsymbol{\xi}+\boldsymbol{\eta}^{H} \overline{\boldsymbol{C}} \boldsymbol{\eta}+1} \leq \rho \frac{\boldsymbol{A} \bullet \boldsymbol{X}_{1}^{\star}+\overline{\boldsymbol{A}} \bullet \boldsymbol{X}_{2}^{\star}}{\boldsymbol{C} \bullet \boldsymbol{X}_{1}^{\star}+\overline{\boldsymbol{C}} \bullet \boldsymbol{X}_{2}^{\star}+1}\right) \\
& \leq\left(\frac{4 \rho}{\omega-2 \rho}\right)^{2} .
\end{aligned}
$$


Proof: We delegate the proof in the the companion technical report [31]. We remark that the upper bounds in (40) and (41) hold simultaneously.

In our problem, we have $\operatorname{rank}\left(\boldsymbol{A}_{k, i}\right)=\operatorname{rank}\left(\overline{\boldsymbol{A}}_{k, i}\right)=1$. Denoting $c_{k, i}=\frac{\min \left\{\boldsymbol{A}_{k, i} \bullet \boldsymbol{W}_{1}^{\star}, \overline{\boldsymbol{A}}_{k, \boldsymbol{}} \bullet \boldsymbol{W}_{2}^{\star}\right\}}{\boldsymbol{A}_{k, i} \bullet \boldsymbol{W}_{1}^{\star}+\overline{\boldsymbol{A}}_{k, i} \bullet \boldsymbol{W}_{2}^{\star}}$, we can apply (40) to bound event $\mathcal{E}_{k, i}$ as $\operatorname{Pr}\left(\mathcal{E}_{k, i}\right) \leq\left(\frac{4 \rho}{c_{k, i}-2 \rho}\right)^{2}, \forall k, i$. Then, by choosing $\rho=\omega /(7 \sqrt{M})$ with $\omega=\min _{k, i}\left\{c_{k, i}\right\}$, we may apply Lemma 1 to obtain

$$
\operatorname{Pr}\left(\bigcup_{\substack{k=1, \ldots, G \\ i=1, \ldots, m_{k}}} \mathcal{E}_{k, i}\right) \leq \sum_{k, i} \operatorname{Pr}\left(\mathcal{E}_{k, i}\right) \leq M\left(\frac{4 \rho}{c_{k, i}-2 \rho}\right)^{2}<\frac{3}{4} .
$$

On the other hand, we can also consider the second upper bound in Lemma 1. By choosing $\rho=1 /(8 M)$ we have

$$
\operatorname{Pr}\left(\bigcup_{\substack{k=1, \ldots, G \\ i=1, \ldots, m_{k}}} \mathcal{E}_{k, i}\right) \leq \sum_{k, i} \operatorname{Pr}\left(\mathcal{E}_{k, i}\right) \leq M \frac{4 \rho}{1-2 \rho}<\frac{3}{4} .
$$

Note that these two bounds hold simultaneously given that we pick different values for $\rho$.

Step 3: We then bound $\operatorname{Pr}\left(\bigcup_{j=1}^{J} \mathcal{F}_{j}\right)$ by Lemma 2 .

Lemma 2 Let $\boldsymbol{D}$ and $\overline{\boldsymbol{D}}$ be Hermitian positive semidefinite matrices and $\boldsymbol{\xi} \sim \mathcal{C N}\left(\mathbf{0}, \boldsymbol{X}_{1}^{\star}\right), \boldsymbol{\eta} \sim$ $\mathcal{C N}\left(\mathbf{0}, \boldsymbol{X}_{2}^{\star}\right)$ be independent random vectors. If $\boldsymbol{D} \bullet \boldsymbol{X}_{1}^{\star}+\overline{\boldsymbol{D}} \bullet \boldsymbol{X}_{2}^{\star}=0$, then $\boldsymbol{\xi}^{H} \boldsymbol{D} \boldsymbol{\xi}+\boldsymbol{\eta}^{H} \overline{\boldsymbol{D}} \boldsymbol{\eta}=0$ almost surely. Otherwise, for any $v \geq 2$, we have

$$
\operatorname{Pr}\left(\boldsymbol{\xi}^{H} \boldsymbol{D} \boldsymbol{\xi}+\boldsymbol{\eta}^{H} \overline{\boldsymbol{D}} \boldsymbol{\eta} \geq v\left(\boldsymbol{D} \bullet \boldsymbol{X}_{1}^{\star}+\overline{\boldsymbol{D}} \bullet \boldsymbol{X}_{2}^{\star}\right)\right) \leq 2 \exp \left(-\frac{v}{2}\right) .
$$

Proof: We delegate the proof in in the the companion technical report [31].

Similar to Step 2, by choosing $v=2 \log (16 J)$, we can bound $\operatorname{Pr}\left(\bigcup_{j=1}^{J} \mathcal{F}_{j}\right)$ as

$$
\operatorname{Pr}\left(\bigcup_{j=1}^{J} \mathcal{F}_{j}\right) \leq \sum_{j=1}^{J} \operatorname{Pr}\left(\mathcal{F}_{j}\right) \leq 2 J \cdot \exp \left(-\frac{v}{2}\right)=\frac{1}{8} .
$$

In this way, we can bound the probability $\mathcal{P}$ as

$$
\mathcal{P} \geq 1-\operatorname{Pr}\left(\bigcup_{\substack{k=1, \ldots, G \\ i=1, \ldots, m_{k}}} \mathcal{E}_{k, i}\right)-\operatorname{Pr}\left(\bigcup_{\ell=0}^{L} \mathcal{F}_{\ell}\right)>\frac{1}{8} .
$$

Step 4: Now it is ready for us to show that based on the fact $\mathcal{P}>0$, the desired approximation bounds can be derived. In particular, letting $\widehat{\boldsymbol{W}}_{1}=\boldsymbol{W}_{1} / v$ and $\widehat{\boldsymbol{W}}_{2}=\boldsymbol{W}_{2} / v$, previous discussions 
imply that with probability at least $1 / 8$, the rank-one solutions $\left(\widehat{\boldsymbol{W}}_{1}, \widehat{\boldsymbol{W}}_{2}\right)$ are feasible for Problem (R2SDR) and we have

$$
\begin{aligned}
\theta\left(\widehat{\boldsymbol{W}}_{1}, \widehat{\boldsymbol{W}}_{2}\right) & =\min _{\substack{k=1, \ldots, G \\
i=1, \ldots, m_{k}}} \frac{\boldsymbol{A}_{k, i} \bullet\left(\boldsymbol{W}_{1} / v\right)+\overline{\boldsymbol{A}}_{k, i} \bullet\left(\boldsymbol{W}_{2} / v\right)}{\boldsymbol{C}_{k, i} \bullet\left(\boldsymbol{W}_{1} / v\right)+\overline{\boldsymbol{C}}_{k, i} \bullet\left(\boldsymbol{W}_{2} / v\right)+1} \\
& \geq \frac{\rho}{v} \cdot \theta\left(\boldsymbol{W}_{1}^{\star}, \boldsymbol{W}_{2}^{\star}\right) .
\end{aligned}
$$

Therefore, we have

$$
\theta\left(\widehat{\boldsymbol{W}}_{1}, \widehat{\boldsymbol{W}}_{2}\right) \geq \frac{\omega}{7 \sqrt{M}(2 \log (16 J)+1)} \cdot \theta\left(\boldsymbol{W}_{1}^{\star}, \boldsymbol{W}_{2}^{\star}\right)
$$

and

$$
\theta\left(\widehat{\boldsymbol{W}}_{1}, \widehat{\boldsymbol{W}}_{2}\right) \geq \frac{1}{8 M(2 \log (16 J)+1)} \cdot \theta\left(\boldsymbol{W}_{1}^{\star}, \boldsymbol{W}_{2}^{\star}\right)
$$

hold simultaneously. Since (42) and (43) are true for each randomization $n \in\{1, \ldots, N\}$, it follows that

$$
\begin{aligned}
& \operatorname{Pr}\left(\left\{\exists n: \theta\left(\widehat{\boldsymbol{w}}_{1}^{n}\left(\widehat{\boldsymbol{w}}_{1}^{n}\right)^{H}, \widehat{\boldsymbol{w}}_{2}^{n}\left(\widehat{\boldsymbol{w}}_{2}^{n}\right)^{H}\right) \geq c \cdot \theta\left(\boldsymbol{W}_{1}^{\star}, \boldsymbol{W}_{2}^{\star}\right)\right\}\right) \\
& \geq 1-(7 / 8)^{N},
\end{aligned}
$$

where $\widehat{\boldsymbol{w}}_{1}^{n}$ and $\widehat{\boldsymbol{w}}_{2}^{n}$ are extracted by eigen-decomposition from rank-one matrices $\widehat{\boldsymbol{W}}_{1}$ and $\widehat{\boldsymbol{W}}_{2}$ at randomization time $n$, and $c$ is defined in Theorem 1. This, together with (31), completes the proof of Theorem 1.

\section{REFERENCES}

[1] J. Chamberland, P. Eedara, and A. Taghavi, "Occupancy estimation using distributed WiFi monitoring," in ITA Workshop 2016, Feb. 2016.

[2] M. N. Tehrani, M. Uysal, and H. Yanikomeroglu, "Device-to-device communication in 5G cellular networks: challenges, solutions, and future directions," IEEE Communications Magazine, vol. 52, no. 5, pp. 86-92, 2014.

[3] T. Hynek, D. Halls, and J. Sykora, "Practical implementation of cloud initialization procedure for wireless physical layer network coding clouds," in 20th European Wireless Conference; Proceedings of European Wireless 2014, May 2014, pp. $1-6$.

[4] Y. Li, T. Wu, P. Hui, D. Jin, and S. Chen, "Social-aware D2D communications: qualitative insights and quantitative analysis," Communications Magazine, IEEE, vol. 52, no. 6, pp. 150-158, 2014.

[5] K. C. et al., "C-RAN: the road toward green RAN, white paper," in China Mobile Research Institute, 2011.

[6] Y. Shi, J. Zhang, and K. Letaief, "Group sparse beamforming for green Cloud-RAN," IEEE Trans. Wireless Commun., May 2014.

[7] V. N. Ha, L. B. Le, and N.-D. Dao, "Cooperative transmission in cloud RAN considering fronthaul capacity and cloud processing constraints," in Proc. IEEE Wireless Communications and Networking Conference (WCNC), 2014. 
[8] S. X. Wu, A. M.-C. So, and W.-K. Ma, "A beamformed Alamouti amplify-and-forward scheme in multigroup multicast cloud-relay networks," in Proc. IEEE Int. Conf. Acoust., Speech, Signal Process. (ICASSP), 2015.

[9] S. X. Wu, Q. Li, W.-K. Ma, and A. M.-C. So, "Stochastic amplify-and-forward schemes for multigroup multicast transmission in a distributed relay network," in Proc. 15th IEEE Int. Workshop Signal Process. Advances in Wireless Commun. (SPAWC), Jun. 2015.

[10] S. X. Wu, Q. Li, A. M.-C.So, and W.-K. Ma, "A stochastic beamformed amplify-and-forward scheme in a multigroup multicast MIMO relay network with per-antenna power constraints," submitted to IEEE Trans. Wireless Commun., 2015, available online at http://arxiv.org/abs/1510.02448

[11] S. Fazeli-Dehkordy, S. Shahbazpanahi, and S. Gazor, "Multiple peer-to-peer communications using a network of relays," IEEE Trans. Signal Process., vol. 57, no. 8, pp. 3053-3062, 2009.

[12] B. K. Chalise and L. Vandendorpe, "MIMO relay design for multipoint-to-multipoint communications with imperfect channel state information,” IEEE Trans. Signal Process., vol. 57, no. 7, pp. 2785-2796, 2009.

[13] B. K. Chalise, L. Vandendorpe, and J. Louveaux, "MIMO relaying for multi-point to multi-point communication in wireless networks," in Computational Advances in Multi-Sensor Adaptive Processing, 2007. CAMPSAP 2007. 2nd IEEE International Workshop on. IEEE, 2007, pp. 217-220.

[14] Z. Ding, W. H. Chin, and K. K. Leung, "Distributed beamforming and power allocation for cooperative networks," IEEE Trans. Wireless Commun., vol. 7, no. 5, pp. 1817-1822, May 2008.

[15] V. Havary-Nassab, S. Shahbazpanahi, A. Grami, and Z.-Q. Luo, "Distributed beamforming for relay networks based on second-order statistics of the channel state information," IEEE Trans. Signal Process., vol. 56, no. 9, pp. 4306-4316, 2008.

[16] I. Jimenez, M. Barrenechea, M. Mendicute, and E. Arruti, "Non-linear precoding approaches for non-regenerative multiuser MIMO relay systems," in Signal Processing Conference (EUSIPCO), 2012 Proceedings of the 20th European. IEEE, 2012, pp. 1399-1403.

[17] K. Gomadam and S. Jafar, "Optimal distributed beamforming in relay networks with common interference," in Global Telecommunications Conference, 2007. GLOBECOM '07. IEEE, Nov 2007, pp. 3868-3872.

[18] M. R. Khandaker and Y. Rong, "Joint transceiver optimization for multiuser MIMO relay communication systems," IEEE Trans. Signal Process., vol. 60, no. 11, pp. 5977-5986, 2012.

[19] R. Muhammad, A. Khandaker, and Y. Rong, "Transceiver optimization for multi-hop MIMO relay multicasting from multiple sources," IEEE Trans. Wireless Commun., vol. 13, no. 9, pp. 5162-5172, Sep. 2014.

[20] Z.-Q. Luo, W.-K. Ma, A. M.-C. So, Y. Ye, and S. Zhang, "Semidefinite relaxation of quadratic optimization problems," IEEE Signal Process. Mag., vol. 27, no. 3, pp. 20-34, May 2010.

[21] T.-H. Chang, Z.-Q. Luo, and C.-Y. Chi, "Approximation bounds for semidefinite relaxation of max-min-fair multicast transmit beamforming problem,” IEEE Trans. Signal Process., vol. 56, no. 8, pp. 3932-3943, 2008.

[22] S. Ji, S. X. Wu, A. M. C. So, and W.-K. Ma, "Multi-group multicast beamforming in cognitive radio networks via ranktwo transmit beamformed Alamouti space-time coding," in Proc. IEEE Int. Conf. Acoust., Speech, and Signal Process. (ICASSP), May 2013, pp. 4409-4413.

[23] S. X. Wu, W.-K. Ma, and A. M. C. So, "Physical-layer multicasting by stochastic transmit beamforming and Alamouti space-time coding," IEEE Trans. Signal Process., vol. 61, no. 17, pp. 4230-4245, Sept. 2013.

[24] A. Schad, K. L. Law, and M. Pesavento, “A convex inner approximation technique for rank-two beamforming in multicasting relay networks," in Proceedings of the 20th European Signal Processing Conference (EUSIPCO), 2012, pp. 1369-1373. 
[25] A. Schad, K. L. Law and M. Pesavento, "Rank-two beamforming and power allocation in multicasting relay networks," IEEE Trans. Signal Process., vol. 63, no. 13, pp. 3435-3447, 2015.

[26] N. Bornhorst, M. Pesavento, and A. B. Gershman, "Distributed beamforming for multi-group multicasting relay networks," IEEE Trans. Signal Process., vol. 60, no. 1, pp. 221-232, 2012.

[27] L.-N. Tran, M. F. Hanif, and M. Juntti, "A conic quadratic programming approach to physical layer multicasting for large-scale antenna arrays," IEEE Signal Process. Lett., vol. 21, no. 1, pp. 114-117, 2014.

[28] D. Christopoulos, S. Chatzinotas, and B. Ottersten, "Multicast multigroup beamforming for per-antenna power constrained large-scale arrays," Proc. of IEEE Int. Work-shop on Signal Process. Advances for Wirel. Commun (SPAWC), Jun. 2015.

[29] O. Mehanna, K. Huang, B. Gopalakrishnan, A. Konar, and N. Sidiropoulos, "Feasible point pursuit and successive approximation of non-convex QCQPs,” IEEE Signal Process. Lett., vol. 22, no. 7, pp. 804-808, 2015.

[30] B. Gopalakrishnan and N. Sidiropoulos, "High performance adaptive algorithms for single-group multicast beamforming," IEEE Trans. Signal Process., vol. 63, no. 16, pp. 4373-4384, Aug 2015.

[31] S. X. Wu, J. Pan, A. M.-C. So, and W.-K. Ma, "Some proof derivations and further simulation results for "Strategies for Exploring Two Degrees-of-freedom in Relay Beamforming Networks and the Performance Analysis"," Technical Report, Department of Systems Engineering and Engineering Management, The Chinese University of Hong Kong, Feb 2016. Available online at http://arxiv.org/abs/1602.06500.

[32] Y. Huang, Q. Li, W.-K. Ma, and S. Zhang, "Robust multicast beamforming for spectrum sharing-based cognitive radios," IEEE Trans. Signal Process., vol. 60, no. 1, pp. 527-533, 2012.

[33] K. T. Phan, S. Vorobyov, N. D. Sidiropoulos, C. Tellambura et al., "Spectrum sharing in wireless networks via qos-aware secondary multicast beamforming," IEEE Transactions on Signal Processing, vol. 57, no. 6, pp. 2323-2335, 2009.

[34] Y. J. Zhang and A. M.-C. So, "Optimal spectrum sharing in mimo cognitive radio networks via semidefinite programming," IEEE Journal on Selected Areas in Communications, vol. 29, no. 2, pp. 362-373, 2011.

[35] E. Karipidis, N. D. Sidiropoulos, and Z.-Q. Luo, "Quality of service and max-min-fair transmit beamforming to multiple co-channel multicast groups,” IEEE Trans. Signal Process., vol. 56, no. 3, pp. 1268-1279, Mar. 2008.

[36] Z.-Q. Luo, N. D. Sidiropoulos, P. Tseng, and S. Zhang, "Approximation bounds for quadratic optimization with homogeneous quadratic constraints," SIAM J. Optim., vol. 18, no. 1, pp. 1-28, 2007.

[37] L. R. Varshney, "Transporting information and energy simultaneously," in Information Theory, 2008. ISIT 2008. IEEE International Symposium on. IEEE, 2008, pp. 1612-1616.

[38] P. Grover and A. Sahai, "Shannon meets tesla: Wireless information and power transfer." in ISIT, 2010, pp. $2363-2367$.

[39] R. Zhang and C. K. Ho, "Mimo broadcasting for simultaneous wireless information and power transfer," Wireless Communications, IEEE Transactions on, vol. 12, no. 5, pp. 1989-2001, 2013.

[40] S. He, Z.-Q. Luo, J. Nie, and S. Zhang, "Semidefinite relaxation bounds for indefinite homogeneous quadratic optimization," SIAM Journal on Optimization, vol. 19, no. 2, pp. 503-523, 2008.

[41] D. Tse and P. Viswanath, Fundamentals of wireless communication. Cambridge university press, 2005.

[42] Y. Huang and S. Zhang, "Complex matrix decomposition and quadratic programming," Math. of Oper. Research, vol. 32, pp. 758-768, 2007.

[43] IEEE Standard for Local and Metropolitan Area Networks, Part 16: Air Interface for Fixed and Mobile Broadband Wireless Access Systems, IEEE Std. 802.16e, 2005. 